\title{
APPROXIMATION RESULTS IN HILBERT CUBE MANIFOLDS
}

\author{
BY
}

T. A. CHAPMAN ${ }^{1}$

\begin{abstract}
The purpose of this paper is to answer questions of the following type for maps $f: M \rightarrow N$ between $Q$-manifolds: When is $f$ close to an approximate fibration? When is $f$ close to a fiber bundle map? When is $f$ close to a block bundle map? For the second and third questions a series of obstructions are encountered in the lower algebraic $K$-theoretic functors $K_{-i}$ of Bass.
\end{abstract}

1. Introduction. Recall that the Hilbert cube $Q$ is the countable infinite product of closed intervals, and a $Q$-manifold is a separable metric manifold modeled on $Q$. The purpose of this paper is to investigate questions of the following sort: If $f$ : $M \rightarrow N$ is a map between $Q$-manifolds, when can $f$ be approximated by "nice" maps? The "nice" maps referred to include homeomorphisms, approximate fibrations, and block bundles. The key idea common to all of these results is a very carefully controlled engulfing. This is established very early in $\$ 3$ by use of $Z$-set unknotting, and is then used at crucial points throughout the paper. This entire line of research is a natural outgrowth of the approximation results of [4], [5], and [14].

With the only exception being some rare instances in which Hurewicz fibrations are introduced for technical reasons, all spaces in this paper will be locally compact, separable, and metric. A map $f: X \rightarrow Y$ (i.e., a continuous function) is proper provided that $f^{-1}(C)$ is compact, for all compact $C \subset Y$. If $\alpha$ is an open cover of $Y$, then a proper map $f: X \rightarrow Y$ is said to be an $\alpha$-fibration (or, $f$ has the $\alpha$-lifting property) if for all maps $F: Z \times[0,1] \rightarrow Y$ and $\tilde{F}_{0}: Z \rightarrow X$ for which $f \tilde{F}_{0}=F_{0}$, there is a map $G: Z \times[0,1] \rightarrow X$ such that $G_{0}=\tilde{F}_{0}$ and $f G$ is $\alpha$-close to $F$. This latter statement means that given any $(z, t) \in Z \times[0,1]$ there is a $U \in \alpha$ containing both $f G(z, t)$ and $F(z, t)$. Finally, a proper map $p: X \rightarrow Y$ is said to be an approximate fibration provided that it has the $\alpha$-lifting property, for all open covers $\alpha$ of $Y$. We refer the reader to [9] where the notion of an approximate fibration was introduced.

Our first result is concerned with the homotopy detection of those maps which are close to approximate fibrations.

THeOREM 1. Let $B$ be an $A N R$ and let $\alpha$ be an open cover of $B$. There exists an open cover $\beta$ of $B$ so that if $M$ is $Q$-manifold and $f: M \rightarrow B$ is a $\beta$-fibration, then $f$ is $\alpha$-close to an approximate fibration.

Received by the editors February 22, 1979 and, in revised form, January 24, 1980.

AMS (MOS) subject classifications (1970). Primary 57E25, 57A20.

Key words and phrases. Hilbert cube manifolds, engulfing, block bundles, aproximate fibration, Whitehead torsion.

${ }^{1}$ Supported in part by NSF Grant MCS 76-06929.

(C) 1980 American Mathematical Society $0002-9947 / 80 / 0000-0550 / \$ 09.00$ 
The proof of this result, which is given in $\$ 6$, first uses some $Q$-manifold results from [6] to reduce it to the case in which $B$ is a polyhedron. Then the proof uses a handle lemma from $\$ 5$ which is established by engulfing and torus geometry.

In [13] a proof is given that any finitely dominated polyhedron can be realized as the fiber of an approximate fibration $p: M \rightarrow S^{1}$, where $M$ is a $Q$-manifold. In the following corollary of Theorem 1 we recover this result. See $\$ 6$ for a proof.

Corollary [13]. If $K$ is a finitely dominated polyhedron, then there is a $Q$-manifold $M$ and an approximate fibration $p: M \rightarrow S^{1}$ whose (homotopy) fiber is $K$.

The following notation will be used throughout this paper. If $\alpha$ is an open cover of $Y$, then a homotopy $h_{t}: X \rightarrow Y$ is said to be an $\alpha$-homotopy provided that each set $\left\{h_{t}(x) \mid 0 \leqslant t \leqslant 1\right\}$ lies in some element of $\alpha$. A proper map $f: X \rightarrow Y$ is an $\alpha$-equivalence provided that there is a proper map $g: Y \rightarrow X$ and proper homotopies $\varphi_{t}: g f \simeq \mathrm{id}_{X}, \theta_{t}: f g \simeq \mathrm{id}_{Y}$ such that $f \varphi_{t}: X \rightarrow Y$ and $\varphi_{t}: Y \rightarrow Y$ are $\alpha$-homotopies. We write this as

$$
\varphi_{t}: g f \stackrel{f^{-1}(\alpha)}{\simeq} \text { id and } \theta_{t}: f g \stackrel{\alpha}{\simeq} \text { id, }
$$

where $f^{-1}(\alpha)$ denotes the open cover of $X$ defined by $f^{-1}(\alpha)=\left\{f^{-1}(U) \mid U \in \alpha\right\}$. Finally if $Y$ has a specified metric and $\varepsilon>0$ is given, then we will also use $\varepsilon$ to denote the open cover of $Y$ by balls of diameter $\varepsilon$. This convention means that we have also defined the notions of $\varepsilon$-homotopy and $\varepsilon$-homotopy equivalence.

Our metric on euclidean $n$-space $R^{n}$ is the one derived from the standard norm, $\|x\|=\left(\left|x_{1}\right|^{2}+\left|x_{2}\right|^{2}+\cdots+\left|x_{n}\right|^{2}\right)^{1 / 2}$. A homotopy $h_{t}: X \rightarrow R^{n}$ is said to be bounded provided that there is a constant $k>0$ so that $h_{t}$ is a $k$-homotopy. If $Y$ is compact, then a proper map $f: X \rightarrow R^{n} \times Y$ is said to be a bounded homotopy equivalence if there is a constant $k>0$ for which $f$ is a $p^{-1}(k)$-equivalence, where $p=$ projection to $R^{n}$.

For any compact $Q$-manifold $F$ let $\delta(F)$ denote the set of equivalence classes of the form [f], where $f: M \rightarrow F$ is a homotopy equivalence ( $M$ is also a compact $Q$-manifold). Another such map, $f^{\prime}: M^{\prime} \rightarrow F$, is defined to be equivalent to $f$ provided that there is a homeomorphism $h: M \rightarrow M^{\prime}$ for which $f^{\prime} h$ is homotopic to $f$. In analogy with the definition of $\delta(F)$ we also define $\mathcal{S}_{b}\left(R^{n} \times F\right)$, where bounded homotopies and bounded homotopy equivalences are used in lieu of homotopies and homotopy equivalences.

For our next result we will first have to recall material from [2, Chapter XII]. For more details see [1] and $\$ 7$ of this paper. If $T^{n}$ is the $n$-torus, then there is a functorial direct sum decomposition of the Whitehead group $\mathrm{Wh}\left(T^{n} \times F\right)$ :

$$
\mathrm{Wh}\left(T^{n} \times F\right)=\mathrm{Wh}(F) \oplus \sum_{i=1}^{n}\left(\begin{array}{c}
n \\
i
\end{array}\right) \tilde{K}_{1-i}(F) \oplus \text { Nil Terms. }
$$

Here Wh is the Whitehead group functor, $\tilde{K}_{0}$ is the reduced projective class group functor, and the $\tilde{K}_{1-i}$ 's are the lower algebraic $K$-theoretic functors of Bass. All the above groups vanish if $\pi_{1}$ of each component of $F$ is free abelian or, more generally, poly $Z$ [12].

THEOREM 2. If $F$ is a compact $Q$-manifold, then $\mathcal{S}_{b}\left(R^{n} \times F\right)$ is in 1-1 correspondence with $\mathrm{Wh}(F)$ for $n=0$, and with $\tilde{K}_{1-n}(F)$ for $n \geqslant 1$. 
The proof of this result, which appears in $\S 8$, only treats the cases $n \geqslant 1$. The case $n=0$ reduces to $\delta(F)$, and it follows from [6, Chapter 12] that this is just $\mathrm{Wh}(F)$.

Our next result is a generalization of the main result of [5] on approximating maps into bundles by homeomorphisms.

TheOREM 3. Let $B$ be an $A N R$ and let $\alpha$ be an open cover of $B$. There exists an open cover $\beta$ of $B$ so that if $M$ and $E$ are $Q$-manifolds, $p: E \rightarrow B$ is a $\beta$-fibration, and $f: M \rightarrow E$ is a $p^{-1}(\beta)$-equivalence, then $f$ is $p^{-1}(\alpha)$-homotopic to a homeomorphism provided that the groups $\mathrm{Wh}(\mathcal{F})$ and $\tilde{K}_{-n}(\mathscr{F})(n \geqslant 0)$ are all zero, where $\mathscr{F}$ is the homotopy fiber of $p^{2}$

Important special cases of this occur when $p: E \rightarrow B$ is (1) a fiber bundle with fiber a compact ANR, (2) an approximate fibration, and (3) a block bundle (see definition below). The proof of this result, which is given in $\$ 9$, first proceeds as in Theorem 1 to a quick reduction to the case in which $B$ is a polyhedron. Then Theorem 2 is used as a "handle lemma" to induct on $\operatorname{dim} B$.

We point out that if $B$ is a polyhedron and $\operatorname{dim} B=n$ in Theorem 3 above, then we only need $\mathrm{Wh}(\mathscr{F})$ and $\tilde{K}_{-i}(\mathscr{F}), 0 \leqslant i \leqslant n-1$, to vanish. The assumption that all of these obstruction groups vanish is an admission of ignorance. The situation is complicated by the fact that the obstructions in $\tilde{K}_{-(i-1)}(\mathscr{F})$ are not defined unless the obstructions in $\tilde{K}_{-i}(\mathscr{F})$ are zero. So a remaining problem is to work out an obstruction theory which gives a general answer to the problem of Theorem 3.

For our last result we will need the following notion. Let $B$ be a polyhedron with a fixed triangulation. A proper map $p$ of a $Q$-manifold $E$ to $B$ is said to be a block bundle with fiber a compact $Q$-manifold $F$ if for each simplex $\sigma$ of $B$ we have a block-preserving homeomorphism $f: p^{-1}(\sigma) \rightarrow \sigma \times F$, i.e., for each face $\tau$ of $\sigma$ we have $f\left(p^{-1}(\tau)\right)=\tau \times F$. In $\S 10$ we prove that if $p: E \rightarrow B$ is a block bundle, then there is a block preserving homotopy of $p$ to an approximate fibration.

Our next result is concerned with the following question: When is a proper map of a $Q$-manifold to a polyhedron close to a block bundle (with respect to some fine subdivision of the polyhedron)? It follows from the paragraph above that one necessary condition for this to happen is that the map must be an $\alpha$-fibration, for $\alpha$ a fine open cover of the polyhedron. Starting with this necessary condition, the following result gives us sufficient conditions for this to happen. As in Theorem 3 it would be nice to have an obstruction theory giving the general answer.

THEOREM 4. Let $B$ be a polyhedron and let $\alpha$ be an open cover of $B$. There exist an open cover $\beta$ of $B$ and a triangulation of $B$ so that if $M$ is a $Q$-manifold and $f$ : $M \rightarrow B$ is a proper map which is a $\beta$-fibration, then $f$ is $\alpha$-homotopic to a block bundle provided that the groups $\mathrm{Wh}(\mathscr{F})$ and $\tilde{K}_{-n}(\mathscr{F})(n \geqslant 0)$ are all zero, where $\mathscr{F}$ is the homotopy fiber of $f$.

\footnotetext{
${ }^{2}$ If $B$ is not connected, then these restrictions are placed on the various homotopy fibers arising from the different components of $B$.
} 
COROllary. Let $B$ be a polyhedron, let $M$ be a $Q$-manifold, and let $f: M \rightarrow B$ be an approximate fibration with homotopy fiber $\mathcal{F}$. Then $f$ can be approximated arbitrarily closely by block bundles provided that $\pi_{1}(\mathscr{F})$ is free abelian.

This result follows quickly from Theorems 1 and 4 provided that we recall the remark made in the paragraph preceding Theorem 2 about the vanishing of the groups $\mathrm{Wh}(F)$ and $\tilde{K}_{-n}(F)$.

Finally we remark that there is an analogue of Theorem 4 for $h$-block bundles, where a proper map $p$ of a $Q$-manifold $M$ to a polyhedron $B$ is said to be an $h$-block bundle with fiber a compact $Q$-manifold $F$ provided that for each simplex $\sigma$ of $B$ there is a block-preserving homotopy equivalence $f: p^{-1}(\sigma) \rightarrow \sigma \times F$. Here is the analogue of Theorem 4 for $h$-block bundles. The proof is left to the interested reader.

THEOREM 4'. Given the data of Theorem 4, $f$ is $\alpha$-homotopic to an h-block bundle provided that the groups $\tilde{K}_{-n}(\mathcal{F})(n \geqslant 0)$ are all zero.

Upon careful examination the reader should observe that the engulfing tricks that we use do have finite-dimensional analogues, and therefore there must be analogues of Theorems 1-4 for topological $n$-manifolds. This is indeed the case and it will be the subject of a future paper.

2. General preliminaries. In this section we will introduce some additional notation and remind the reader of some well-known results from the literature which will be used throughout this paper.

Recall from $\$ 1$ that $R^{n}$ denotes euclidean $n$-space, i.e., $R^{n}=R \times \cdots \times R(n$ times). In $R^{n}$ we consider $n$-cells of the form $B_{r}^{n}=[-r, r]^{n}$. We use $S^{1}$ to denote the set of complex numbers of absolute value 1 , and the $n$-torus is $T^{n}=S^{1}$ $\times \cdots \times S^{1}$. We always let $I=[0,1]$ and the standard $n$-cell is $I^{n}=[0,1]$ $\times \cdots \times[0,1]$. The Hilbert cube $Q$ will be the countable product $Q=I \times I$ $\times \ldots$ so that for each $n$ we have an internal factorization $Q=I^{n} \times Q_{n+1}$. Note that if $n$ is large, then each set $\{x\} \times Q_{n+1}$ has a small diameter. It will be convenient to regard $I^{n}$ as identified with the subset $I^{n} \times\{(0,0, \ldots)\}$ of $Q$; thus $I^{n} \subset Q$.

Expanding on the definition of an $\alpha$-equivalence which was given in $\S 1$ let $f$ : $X \rightarrow Y$ be proper, let $C \subset Y$ be closed, and let $\alpha$ be an open cover of $Y$. Then $f$ is said to be an $\alpha$-equivalence over $C$ if there is a proper map $g: C \rightarrow X$ and proper homotopies $\varphi_{t}: g f \mid f^{-1}(C) \simeq^{f^{-1}(\alpha)}$ id, $\theta_{t}: f g \simeq^{\alpha}$ id. This means that $\varphi_{t}: f^{-1}(C) \rightarrow X$ and $\theta_{t}: C \rightarrow Y$ are proper homotopies such that $\varphi_{0}=g f \mid f^{-1}(C), \varphi_{1}$ is the inclusion $f^{-1}(C) \hookrightarrow X, \varphi_{t}$ is an $f^{-1}(\alpha)$-homotopy, $\theta_{0}=f g, \theta_{1}$ is the inclusion $C \hookrightarrow Y$, and $\theta_{t}$ is an $\alpha$-homotopy. Note that if $C \subset G \subset Y$, where $C$ is closed and $G$ is open, then for all sufficiently fine open covers $\alpha$ of $Y$ any $\alpha$-equivalence $f: X \rightarrow Y$ restricts to an $(\alpha \cap G)$-equivalence over $C, f \mid f^{-1}(G): f^{-1}(G) \rightarrow G$. (Here $\alpha \cap G=\{U \cap$ $G \mid U \in \alpha\}$.)

The following result tells us how to detect $\alpha$-equivalences locally. It is essentially a souped-up version of Proposition 3.2 of [4]. 
Proposition 2.1. Let $B$ be a space and let $\gamma$ be an open cover of $B$. For every open cover $\alpha$ of $B$ there exists an open cover $\beta$ of $B$ so that if $f: X \rightarrow Y$ and $p: Y \rightarrow B$ are proper maps such that $X, Y$ are $A N R$ 's and $f$ is $a p^{-1}(\beta)$-equivalence over the closure of each element of $\gamma$, then $f$ is $a p^{-1}(\alpha)$-equivalence.

Proof. We begin by recalling how Proposition 3.2 of [4] applies here. Let $A_{1}, A_{2}$ be closed subsets of $B$ with closed neighborhoods $\tilde{A}_{1}, \tilde{A}_{2}$, respectively. Then Proposition 3.2 of [4] says that for every open cover $\alpha$ of $B$ there is an open cover $\beta$ of $B$ so that if $f: X \rightarrow Y$ is a $p^{-1}(\beta)$-equivalence over $\tilde{A}_{1}$ and $\tilde{A}_{2}$, then $f$ is a $p^{-1}(\alpha)$-equivalence over $A_{1} \cup A_{2}$.

If $B$ is compact then we may assume that $\gamma=\left\{U_{i}\right\}_{i=1}^{n}$ is a finite open cover of $B$. Choose a sequence $\left\{\gamma_{k}\right\}_{k=1}^{n-1}$ of open covers of $B$ of the form $\gamma_{k}=\left\{U_{k, i}\right\}_{i=1}^{n}$ so that $\bar{U}_{1, i} \subset U_{i}$ and $\bar{U}_{k+1, i} \subset U_{k, i}$ (we use ${ }^{-}$to denote closure). We are given that $f$ is a $p^{-1}(\beta)$-equivalence over each $\bar{U}_{i}$. Using Proposition 3.2 of [4], $f$ must be a $p^{-1}\left(\beta_{1}\right)$-equivalence over $\overline{U_{1,1} \cup U_{1,2}}$ and $\bar{U}_{1, i}$, for $3 \leqslant i \leqslant n$, where $\beta_{1}$ can be made as fine as we want corresponding to a fine choice of $\beta$. Again using Proposition 3.2 of [4], $f$ must be a $p^{-1}\left(\beta_{2}\right)$-equivalence over the closure of $U_{2,1} \cup U_{2,2} \cup U_{2,3}$ and $\bar{U}_{2, i}, 4 \leqslant i \leqslant n$, where $\beta_{2}$ can be made as fine as we want corresponding to a fine choice of $\beta_{1}$. Continuing this we finally get that $f$ is a $p^{-1}(\alpha)$-equivalence over the closure of $\cup_{i=1}^{n} U_{n-1, i}=B$.

If $B$ is not compact (and therefore locally compact) we can write $B=\cup_{i=1}^{\infty} B_{i}$, where $B_{i}$ is compact and $B_{i} \cap B_{j}=\varnothing$ for $|i-j| \geqslant 2$. Let $\tilde{B}_{i}$ be a closed neighborhood of $B_{i}$ so that we also have $\tilde{B}_{i} \cap \tilde{B}_{j}=\varnothing$ for $|i-j| \geqslant 2$. By the ideas used in the compact case we conclude that $f$ is a $p^{-1}\left(\alpha^{\prime}\right)$-equivalence over each $\tilde{B}_{i}$, where $\alpha^{\prime}$ can be made as fine as we want corresponding to a fine choice of $\beta$. Clearly $f$ is also a $p^{-1}\left(\alpha^{\prime}\right)$-equivalence over $B^{\prime}=\bigcup\left\{\tilde{B}_{i} \mid i\right.$ odd $\}$ and $B^{\prime \prime}=\bigcup\left\{\tilde{B}_{i} \mid i\right.$ even $\}$. By Proposition 3.2 of [4] we conclude that $f$ is a $p^{-1}(\alpha)$-equivalence over the union of $\cup\left\{B_{i} \mid i\right.$ odd $\}$ and $\cup\left\{B_{i} \mid i\right.$ even $\}$.

REMARKS. 1. There is a similarly-worded local version of this result whose conclusion states that $f$ is a $p^{-1}(\alpha)$-equivalence over some closed $C \subset Y$. The proof again follows quickly from Proposition 3.2 of [4].

2. The above proof works if we merely assume that $X, Y$ are separable metric ANR's and $f, p$ are no longer necessarily proper. For this to make sense we also have to drop the requirement that the map $g$ and homotopies $\varphi_{t}, \theta_{t}$ of the definition are proper.

We now expand on the definition of an $\alpha$-fibration which was given in $\S 1$. Let $f$ : $X \rightarrow Y$ be proper, let $C \subset Y$ be closed, and let $\alpha$ be an open cover of $Y$. Then $f$ is said to be an $\alpha$-fibration over $C$ if for all maps $F: Z \times I \rightarrow C$ and $\tilde{F}_{0}: Z \rightarrow X$ for which $f \tilde{F}_{0}=F_{0}$, there is a map $G: Z \times I \rightarrow X$ such that $G_{0}=\tilde{F}_{0}$ and $f G$ is $\alpha$-close to $F$. Note that if $f: X \rightarrow Y$ is an $\alpha$-fibration and $C \subset G \subset Y$, where $C$ is closed and $G$ is open, then the restriction $f \mid f^{-1}(G): f^{-1}(G) \rightarrow G$ is an $(\alpha \cap G)$-fibration over $C$ provided that $\alpha$ is sufficiently fine.

Here is an analogue of Proposition 2.1 for $\alpha$-fibrations.

Proposition 2.2. Let $B$ be an $A N R$ and let $\gamma$ be an open cover of B. For every 
open cover $\alpha$ of $B$ there exists an open cover $\beta$ of $B$ so that if $X$ is an $A N R$ and $f$ : $X \rightarrow B$ is a $\beta$-fibration over the closure of each element of $\gamma$, then $f$ is an $\alpha$-fibration.

Proof. Inspired by the proof of Proposition 2.1, we establish the analogue of Proposition 3.2 of [4]; the proof then follows that of Proposition 2.1. Choose closed subsets $A_{1}, A_{2}$ of $B$ with closed neighborhoods $\tilde{A}_{1}, \tilde{A}_{2}$ respectively, and let $\alpha$ be an open cover of $B$. We want to prove that there exists an open cover $\beta$ of $B$ so that if $f: X \rightarrow B$ is a proper map which is a $\beta$-fibration over $\tilde{A}_{1}$ and $\tilde{A}_{2}$, then $f$ is an $\alpha$-fibration over $A_{1} \cup A_{2}$. Let $F: Z \times I \rightarrow A_{1} \cup A_{2}$ and $\tilde{F}_{0}: \dot{Z} \rightarrow X$ be given so that $f \tilde{F}_{0}=F_{0}$. For simplicity we will only treat the case in which $Z$ is compact. The general (locally compact) case then easily follows from the same ideas.

Our first task will be to treat a special situation which will be used repeatedly to obtain our desired result. Choose open sets $N_{i} \subset B$ so that $A_{i} \subset N_{i}$ and $\bar{N}_{i} \subset \tilde{A}_{i}$, and let $\alpha^{\prime}$ be an open cover of $B$.

Assertion. We can choose the open cover $\beta$ so that if each $F(\{z\} \times I)$ lies in either $N_{1}$ or $N_{2}$, then there is a map $G: Z \times I \rightarrow X$ which extends $\tilde{F}_{0}$ and for which $f G$ is $\alpha^{\prime}$-close to $F$.

Proof. Let $Z_{i}=\left\{z \in Z \mid F(\{z\} \times I) \subset N_{i}\right\}$ and use the fact that $f$ is a $\beta$-fibration over $\tilde{A}_{1}$ to choose a map $G^{1}: Z_{1} \times I \rightarrow X$ so that $G_{0}^{1}=\tilde{F}_{0} \mid Z_{1}$ and so that $f G^{1}$ is $\beta$-close to $F \mid Z_{1} \times I$. We will need the Estimated Homotopy Extension Theorem of [4]. It states that an $\alpha$-homotopy of a closed subset of a metric space into an ANR, $A$, extends to an $\alpha$-homotopy of the entire metric space to $A$ provided that the 0 -level extends. Using this we can adjust $F$ slightly rel $Z \times\{0\}$ so that $F \mid Z_{1} \times I=$ $f G^{1}$. (We also have to cut down the domain of $G^{1}$ slightly.)

Since $Z_{1}$ and $Z_{2}$ are open we can find compacta $C_{i} \subset Z_{i}$ so that $Z=C_{1} \cup C_{2}$. Now let $R_{t}: Z_{2} \times I \rightarrow Z_{2} \times I$ be a homotopy, $0 \leqslant t \leqslant 1$, which affects only the $I$-coordinate, so that

(1) $R_{0}\left(Z_{2} \times I\right) \subset\left(Z_{2} \times\{0\}\right) \cup\left(\left(Z_{1} \cap Z_{2}\right) \times I\right)$,

(2) $R_{t}=$ id on $\left(Z_{2} \times\{0\}\right) \cup\left(\left(C_{1} \cap Z_{2}\right) \times I\right)$, for all $t$,

(3) $R_{1}=$ id.

Let $R:\left(Z_{2} \times I\right) \times[0,1] \rightarrow Z_{2} \times I$ be defined by $R(z, s, t)=R_{t}(z, s)$ and note that $F R_{0}: Z_{2} \times I \rightarrow \tilde{A}_{2}$ lifts to the map $g R_{0}: Z_{2} \times I \rightarrow X$, where $g:\left(Z_{2} \times\{0\}\right) \cup\left(\left(Z_{1}\right.\right.$ $\left.\left.\cap Z_{2}\right) \times I\right) \rightarrow X$ is defined by $g=\tilde{F}_{0}$ on $Z_{2} \times\{0\}$ and $g=G^{1}$ on $\left(Z_{1} \cap Z_{2}\right) \times I$. Since $f$ is a $\beta$-fibration over $\tilde{A}_{2}$ we can find a map $H:\left(Z_{2} \times I\right) \times[0,1] \rightarrow X$ so that $H_{0}=g R_{0}$ and so that $f H$ is $\beta$-close to $F R$. The restriction of $H_{1}: Z_{2} \times I \rightarrow X$ to $\left(Z_{2} \times\{0\}\right) \cup\left(\left(C_{1} \cap Z_{2}\right) \times I\right)$ is easily seen to be $f^{-1}(\beta)$-homotopic to the restriction of $g$ to $\left(Z_{2} \times\{0\}\right) \cup\left(\left(C_{1} \cap Z_{2}\right) \times I\right)$ by using the [0,1]-coordinate. Thus by the Estimated Homotopy Extension Theorem there is an $f^{-1}(\beta)$-homotopy of $H_{1}$ to $G^{2}: Z_{2} \times I \rightarrow X$ so that $G_{0}^{2}=\tilde{F}_{0} \mid Z_{2}$ and so that $G^{2}=G^{1}$ on $C_{1} \cap Z_{2}$. Then $G^{1}$ and $G^{2}$ piece together to give our desired map $G: Z \times I \rightarrow X$.

Now returning to the proof of Proposition 2.2 choose a partition of $I, 0=t_{0}<t_{1}$ $<\cdots<t_{n}=1$, so that for each $z \in Z$ and $i$ we have $F\left(\{z\} \times\left[t_{i-1}, t_{i}\right]\right)$ close to $A_{1}$ or $A_{2}$. For each $i$ we will construct a map $G^{i}: Z \times\left[0, t_{i}\right] \rightarrow X$ so that

(1) $G_{0}^{i}=\tilde{F}_{0}$, 
(2) $f G^{i} \mid Z \times\left\{t_{i}\right\}$ is $\alpha^{\prime}$-close to $F \mid Z \times\left\{t_{i}\right\}$,

(3) $f G^{i}$ is $\alpha$-close to $F \mid Z \times\left[0, t_{i}\right]$.

Then $G^{n}: Z \times I \rightarrow X$ will be our desired extension of $\tilde{F}_{0}$.

The case $i=0$ is trivial, so proceeding inductively let $G^{i}: Z \times\left[0, t_{i}\right] \rightarrow X$ be given with the properties listed above. We will construct $G^{i+1}: Z \times\left[0, t_{i+1}\right] \rightarrow X$. Since our $G^{i+1}$ will be an extension of $G^{i}$ we only have to worry about its definition on $Z \times\left[t_{i}, t_{i+1}\right]$. By the Estimated Homotopy Extension Theorem we can adjust $F \mid Z \times\left[t_{i}, t_{i+1}\right]$ slightly to obtain $F^{\prime}: Z \times\left[t_{i}, t_{i+1}\right] \rightarrow B$ so that $F_{t_{i}}^{\prime}=f G_{t_{i}}^{i}$ and so that $F_{t_{i+1}}^{\prime}=F_{t_{i+1}}$. Then we still must have $F^{\prime}\left(\{z\} \times\left[t_{i}, t_{i+1}\right]\right)$ close to $A_{1}$ or $A_{2}$, for all $z$. By the Assertion there is a map $G^{i+1}: Z \times\left[t_{i}, t_{t+1}\right] \rightarrow X$ for which $G_{t_{i}}^{i+1}=G_{t_{i}}^{i}$ and for which $f G^{i+1}$ is $\alpha^{\prime}$-close to $F^{\prime}$. If $\alpha^{\prime}$ is appropriately chosen, then $f G^{i+1}$ is $\alpha$-close to $F \mid Z \times\left[t_{i}, t_{i+1}\right]$. This completes the inductive step.

Remark. As in Proposition 2.1 there is a similarly-worded local version of this result whose conclusion states that $f$ is an $\alpha$-fibration over some closed set $C \subset B$.

The following result gives us another point of view from which we can recognize $p^{-1}(\alpha)$-equivalences.

Proposition 2.3. Let $B$ be an $A N R$ and let $\alpha$ be an open cover of $B$. There exists an open cover $\beta$ of $B$ so that if $p: \mathcal{E} \rightarrow B$ is a Hurewicz fibration, $X$ is arbitrary and $f: X \rightarrow \mathcal{E}$ is a homotopy equivalence for which $p f: X \rightarrow B$ is a $\beta$-fibration, then $f$ is $a$ $p^{-1}(\alpha)$-equivalence.

Proof. As in Remark 2 following Proposition 2.1 we allow that $X$, $\mathcal{E}$ be arbitrary separable metric, and the maps are possibly nonproper. Suppose first that $p f: X \rightarrow B$ is also a Hurewicz fibration. Then $f$ is a fiber preserving map between two Hurewicz fibrations and it is also a homotopy equivalence. In [10, Theorem 6.1] there is a proof that $f$ must be a fiber homotopy equivalence. This means that there is a fiber preserving map $g: \mathcal{E} \rightarrow X$ and fiber preserving homotopies $f g \simeq$ id, $g f \simeq \mathrm{id}$. Therefore $f$ is a $p^{-1}(\alpha)$-equivalence for all open covers $\alpha$ of $B$. This special case does not require that $B$ be a ANR.

The proof given in [10] requires only the homotopy lifting property, and so a slight modification of it works if we merely assume that $p f: X \rightarrow B$ has the $\beta$-lifting property. Let $g: \mathcal{E} \rightarrow X$ be an ordinary homotopy inverse of $f$ and let $h_{t}: f g \simeq \mathrm{id}_{\mathcal{E}}$ be a homotopy. Then the homotopy $p h_{t}:(p f) g \simeq p$ can be $\beta$-lifted to a homotopy $k_{t}: g \simeq g^{\prime}$. The map $g^{\prime}: \mathcal{E} \rightarrow X$ is nearly fiber preserving, and it is our candidate for a $p^{-1}(\alpha)$-inverse of $f$. The argument of [10] quickly adapts to prove that this is the case. The assumption that $B$ be an ANR is needed to construct homotopies between maps to $B$ which are sufficiently close together. Further details are left to the reader.

We write " $f=g$ over $C$ " to mean that $f^{-1}(C)=g^{-1}(C)$ and $f=g$ on $f^{-1}(C)$. In general we say that $f$ has property $\mathbf{P}$ over $C$ whenever $f \mid f^{-1}(C)$ has property $\mathbf{P}$. In some instances when the meaning is clear we will simply write $f$ when the restriction $f \mid$ is intended. Finally, a homotopy $f_{t}: X \rightarrow Y$ is said to be an $\alpha$-homotopy over $C \subset Y$ if $f_{t} \mid f_{0}^{-1}(C): f_{0}^{-1}(C) \rightarrow Y$ is an $\alpha$-homotopy.

The concept of the mapping cylinder will be useful to us. If $f: X \rightarrow Y$ is a map 
between compact spaces, then the mapping cylinder of $f$ is the quotient space

$$
M(f)=(X \times[0,1]) \amalg Y / \sim,
$$

where $\sim$ is the equivalence relation generated by $(x, 1) \sim f(x)$. We identify $X$ with $X \times\{0\} \subset M(f)$ and call it the top. The base of $M(f)$ is the natural copy of $Y$ embedded in it. The collapse of $M(f)$ to its base, $c: M(f) \rightarrow Y$, is the map obtained by retracting each ray $(\{x\} \times[0,1]) \amalg\{f(x)\} / \sim$ to $f(x)$. Generally it is convenient to write $M(f)=(X \times[0,1)) \cup Y$, with the appropriate topology being understood.

In this paper we will need several standard geometric results from $Q$-manifold theory, and a good reference for all of these is [6]. In particular, the reader should be familiar with $Z$-set unknotting, stability, the Triangulation Theorem, and Edwards' ANR Theorem. There is a slightly stronger form of $Z$-set unknotting which we will need, so we supply a proof.

Proposition 2.4. Let $M_{1}, M_{2}$ be $Q$-manifolds, let $A_{i} \subset M_{i}$ be a $Z$-set for $i=1,2$, and let $u: M_{1} \rightarrow M_{2}$ be a proper map such that $u \mid A_{1}: A_{1} \rightarrow A_{2}$ is a homeomorphism. If there is a proper $\alpha$-homotopy of $u$ to a homeomorphism $h: M_{1} \rightarrow M_{2}$, then there is a homeomorphism $h^{\prime}: M_{1} \rightarrow M_{2}$ for which $h^{\prime}=u$ on $A_{1}$ and there is a proper $\operatorname{St}(\alpha)$-homotopy $u \simeq h^{\prime}$ rel $A_{1}$.

Proof. First we define the open cover $\operatorname{St}(\alpha)$ of $M_{2}$ :

$$
\operatorname{St}(\alpha)=\{\cup\{W \mid W \in \alpha \text { and } W \cap U \neq \varnothing\} \mid U \in \alpha\} .
$$

Let $u_{t}$ denote the proper homotopy $u \simeq h$. We may assume that $h\left(A_{1}\right) \cap A_{2}=\varnothing$ and we can adjust $u_{t}$ slightly so that $u_{t} \mid: A_{1} \rightarrow M_{2}$ is an $\sigma$-isotopy of $Z$-embeddings whose various levels are pairwise disjoint. Now let $g_{t}: M_{2} \rightarrow M_{2}$ be an $\alpha$-isotopy for which $g_{0}=$ id and $g_{t}=u_{t} u^{-1}$ on $u\left(A_{1}\right)$. The existence of $g_{t}$ follows easily from the proof of 19.4 of [6], but apparently not from the statement. Then $h^{\prime}=g_{1}^{-1} h$ : $M_{1} \rightarrow M_{2}$ is a homeomorphism which is $\operatorname{St}(\alpha)$-homotopic to $u$ rel $A_{1}$ via the homotopy $g_{t}^{-1} u_{t}: u \simeq g_{1}^{-1} h$.

REMARK. There is a similarly-worded version of this result in which the homeomorphism $h$ is replaced by an open embedding. This particular version is used near the end of $\S 8$.

There is a large collection of results from simple-homotopy theory which we will need. An excellent reference is [8]. Also the reader should be aware of the Classification Theorem of $[6$, p. 86], which is the main connection between $Q$-manifold theory and simple-homotopy theory.

Finally, we will need the finiteness obstruction of Wall [18], which assigns to each finitely dominated space $X$ an element $\sigma(X)$ of the reduced projective class group $\tilde{K}_{0} \pi_{1}(X)=\tilde{K}_{0}(X)$. This element $\sigma(X)$ is a homotopy-type invariant and it vanishes iff $X$ is homotopy equivalent to a compact polyhedron.

3. Engulfing. The purpose of this section is to establish Theorem 3.3, which is the main engulfing result for $Q$-manifolds which will be needed in the sequel. In Lemma 3.1 we use nothing more complicated than $Z$-set unknotting to establish the basic engulfing result. This is then used to establish Lemma 3.2, which turns 
out to be a global version of Theorem 3.3. Finally, Theorem 3.3 follows as a corollary to the proofs of Lemmas 3.1 and 3.2. For notation $X$ will be a compact metric space with a specified metric for Lemmas 3.1 and 3.2.

LeMma 3.1. For every $\varepsilon>0$ there exists $a \delta>0$ so that if $M$ is a $Q$-manifold and $f: M \rightarrow X \times R$ is a $\delta$-fibration over $X \times[-3,3]$, then there exists a homeomorphism $h: M \rightarrow M$ for which

$$
f^{-1}(X \times(-\infty, 1]) \subset h f^{-1}(X \times(-\infty, 0)) .
$$

Moreover if $p_{1}: X \times R \rightarrow X$ is projection, then there is a homotopy $h_{t}: \mathrm{id}_{M} \simeq h$ which is supported on $f^{-1}(X \times[-2,2])$ and which is a $\left(p_{1} f\right)^{-1}(\varepsilon)$-homotopy.

Proof. Since proj: $M \times[0,1] \rightarrow M$ is a near homeomorphism we may replace $M$ by $M \times[0,1]$ and regard each $f(\{m\} \times[0,1])$ as having small diameter. The first step is to obtain an isotopy $u_{t}: M \times[0,1] \rightarrow M \times[0,1]$ such that $u_{0}=\mathrm{id}, u_{t}$ is supported on $f^{-1}(X \times[-1.5,1.5]), u_{t}$ is a $\left(p_{1} f\right)^{-1}(\delta)$-homotopy, and

$$
(M \times\{0\}) \cap f^{-1}(X \times(-\infty, 1.4]) \subset u_{1} f^{-1}(X \times(-\infty, 0)) .
$$

One constructs $u_{t}$ as the inverse of an isotopy whose 1-level takes $(M \times\{0\}) \cap$ $f^{-1}(X \times(-\infty, 1.4])$ into $f^{-1}(X \times(-\infty, 0))$. This isotopy comes immediately from $Z$-set unknotting, and the homotopy needed to apply the $Z$-set unknotting theorem comes from the definition of a $\delta$-fibration.

The next step is to again use $Z$-set unknotting to find an isotopy $v_{t}: M \times[0,1]$ $\rightarrow M \times[0,1]$ such that $v_{0}=\mathrm{id}, v_{t}$ is supported on $f^{-1}(X \times[-1.6,1.3]), v_{t}$ is a $\left(p_{1} f\right)^{-1}(\delta)$-homotopy, and

$$
(M \times\{1\}) \cap f^{-1}(X \times[-1.5, \infty)) \subset v_{1}^{-1} f^{-1}(X \times(1, \infty)) .
$$

The construction of $v_{t}$ is similar to the construction of $u_{t}$.

The last step is to find an isotopy $w_{t}: M \times[0,1] \rightarrow M \times[0,1]$ such that $w_{0}=\mathrm{id}$, $w_{t}$ is supported on $f^{-1}(X \times[-1.5,1.5]), w_{1} u_{1} f^{-1}(X \times[0, \infty))$ is close to the set $f^{-1}(X \times[1.4, \infty)) \cup\left[(M \times\{1\}) \cap f^{-1}(X \times[-1.5, \infty))\right]$, and $w_{t}$ is a $\left(p_{1} f\right)^{-1}(\delta)-$ homotopy. We can easily construct $w_{t}$ by moving along the $[0,1]$-factor. Then $h_{t}=v_{t} w_{t} u_{t}$ and $h=h_{1}$ fulfill our requirements. (The confused reader should draw a picture.)

We now establish a generalization of Lemma 3.1. For notation let $\theta: R \rightarrow R$ be a homeomorphism which is supported on $[-1,1]$.

LEMMA 3.2. For every $\varepsilon>0$ there exists a $\delta>0$ so that if $f: M \rightarrow X \times R$ is as in Lemma 3.1, then there exists a homeomorphism $\tilde{\theta}: M \rightarrow M$ which is supported on $f^{-1}(X \times[-2,2])$ and for which $d\left(f \tilde{\theta},\left(\mathrm{id}_{X} \times \theta\right) f\right)<\varepsilon$. Moreover we can construct $\tilde{\theta}$ so that there is a $\left(p_{1} f\right)^{-1}(\varepsilon)$-homotopy of $\tilde{\theta}$ to id.

Proof. We start by choosing a fine partition of $[-1,1],-1=x_{0}<x_{1}$ $<\cdots<x_{n-1}<x_{n}=1$. We will use Lemma 3.1 to construct a "stacking," i.e., a homeomorphism $\tilde{\theta}: M \rightarrow M$ which is supported on $f^{-1}(X \times[-2,2])$, which satisfies $d\left(p_{1} f \tilde{\theta}, p_{1} f\right)<\varepsilon / 2$, and which also satisfies

$$
f^{-1}\left(X \times\left(-\infty, \theta\left(x_{i-1}\right)\right]\right) \subset \tilde{\theta} f^{-1}\left(X \times\left(-\infty, x_{i}\right]\right) \subset f^{-1}\left(X \times\left(-\infty, \theta\left(x_{i}\right)\right]\right),
$$


for $1 \leqslant i \leqslant n-1$. If the support of $\tilde{\theta}$ is sufficiently close to $f^{-1}(X \times[-1,1])$, and if the partition $\left\{x_{i}\right\}$ is sufficiently fine, then we will clearly have $d(f \tilde{\theta}$, (id $\left.\times \theta) f\right)<$ $\varepsilon$. The homotopy of $\tilde{\theta}$ to id will be clear from the construction. We will actually construct $\tilde{\theta}$ as a composition, $\tilde{\theta}=\tilde{\theta}_{n-1} \circ \cdots \circ \tilde{\theta}_{1}$. The working procedure is to assume that $\delta$ is small enough to perform all of the ensuing constructions. Just to make sure that there is no confusion, here is the order of choices: First the partition $\left\{x_{i}\right\}$ is chosen, then $\delta$ is calculated in terms of this choice.

We start by constructing $\tilde{\theta}_{1}$. By Lemma 3.1 there is a homeomorphism $\tilde{\boldsymbol{\theta}}_{1}$ : $M \rightarrow M$ which is supported on $f^{-1}(X \times[-1,1])$, for which $d\left(p_{1} f \tilde{\theta}_{1}, p_{1} f\right)$ is small, and for which

$$
f^{-1}(X \times(-\infty,-1]) \subset \tilde{\theta_{1}} f^{-1}\left(X \times\left(-\infty, x_{1}\right]\right) \subset f^{-1}\left(X \times\left(-\infty, \theta\left(x_{1}\right)\right]\right) .
$$

Note that the first inclusion will be automatically satisfied provided that $\tilde{\theta}_{1}$ is supported on $f^{-1}(X \times[-1,1])$.

We will construct $\tilde{\theta}_{2}$ as a composition, $\tilde{\theta}_{2}=\theta_{2}^{\prime \prime} \circ \theta_{2}^{\prime}$. It will be supported on $\tilde{\theta}_{1} f^{-1}\left(X \times\left[x_{1}, 1\right]\right)$, so the inclusion (*) will be automatically preserved. The homeomorphism $\theta_{2}^{\prime}$ comes from Lemma 3.1. It is supported on $\tilde{\theta}_{1} f^{-1}\left(X \times\left[x_{1}, 1\right]\right)$ so that $d\left(p_{1} f \theta_{2}^{\prime}, p_{1} f\right)$ is small and so that

$$
f^{-1}\left(X \times\left(-\infty, \theta\left(x_{1}\right)\right]\right) \subset \theta_{2}^{\prime} \tilde{\theta}_{1} f^{-1}\left(X \times\left(-\infty, x_{2}\right]\right) .
$$

The homeomorphism $\theta_{2}^{\prime \prime}$ also comes from Lemma 3.1. It is supported on $f^{-1}\left(X \times\left[\theta\left(x_{1}\right), 1\right]\right)$ (thus preserving the above inclusion) so that $d\left(p_{1} f \theta_{2}^{\prime \prime}, p_{1} f\right)$ is small and so that $\theta_{2}^{\prime \prime} \theta_{2}^{\prime} \tilde{\theta}_{1} f^{-1}\left(X \times\left(-\infty, x_{2}\right]\right) \subset f^{-1}\left(X \times\left(-\infty, \theta\left(x_{2}\right)\right]\right)$. Putting together these two inclusions we have

$f^{-1}\left(X \times\left(-\infty, \theta\left(x_{1}\right)\right]\right) \subset \theta_{2}^{\prime \prime} \theta_{2}^{\prime} \tilde{\theta}_{1} f^{-1}\left(X \times\left(-\infty, x_{2}\right]\right) \subset f^{-1}\left(X \times\left(-\infty, \theta\left(x_{2}\right)\right]\right)$.

Therefore $\tilde{\theta}_{2}=\theta_{2}^{\prime \prime} \circ \theta_{2}^{\prime}$ fulfills our requirements. ${ }^{3}$

It is now clear that we can inductively continue this process to construct our desired $\tilde{\theta}_{i}, 1 \leqslant i \leqslant n-1$.

We are now ready for our main result. For notation let $X$ be a space with a specified metric, let $\varphi: X \rightarrow[0, \infty)$ be proper, and for each $t \in[0, \infty)$ let $X_{t}=$ $\varphi^{-1}([0, t])$. Let $\theta_{t}: R \rightarrow R$ be an isotopy, $t \in[0, \infty)$, which is supported on $[-1,1]$ and for which $\theta_{t}=\mathrm{id}$ for $t \geqslant 1$. This induces a homeomorphism $\theta: X \times R \rightarrow X \times$ $R$ defined by $\theta(x, r)=\left(x, \theta_{\varphi(x)}(r)\right)$. It is supported on $X_{1} \times[-1,1]$.

TheOREM 3.3. For every $\varepsilon>0$ there exists $a \delta>0$ such that if $M$ is a $Q$-manifold and $f: M \rightarrow X \times R$ is a $\delta$-fibration over $X_{3} \times[-3,3]$, then there exists a homeomorphism $\tilde{\theta}: \quad M \rightarrow M$ which is supported on $f^{-1}\left(X_{2} \times[-2,2]\right)$ and which satisfies $d(f \tilde{\theta}, \theta f)<\varepsilon$. Moreover, for every $\mu>0$ there exists $a \nu>0$ such that if $f$ is additionally given to be a $\nu$-fibration over $\left(X_{3}-\dot{X}_{1 / 3}\right) \times[-3,3]$, then the homeomorphism $\tilde{\theta}: M \rightarrow M$ additionally satisfies $d(\tilde{f} \tilde{\theta}, \theta f)<\mu$ over $\left(X-\dot{X}_{2 / 3}\right) \times R$. Also (as in Lemma 3.2), there is a homotopy of $\tilde{\theta}$ to id which is supported on

\footnotetext{
${ }^{3}$ It should be apparent to the reader that each time any of these engulfing moves is constructed, we must actually engulf a little more than is indicated in the above inclusions so that the process can be continued at the next stage.
} 
$f^{-1}\left(X_{2} \times[-2,2]\right)$, which is a $\left(p_{1} f\right)^{-1}(\varepsilon)$-homotopy (globally), and a $\left(p_{1} f\right)^{-1}(\mu)$-homotopy over $\left(X-\dot{X}_{2 / 3}\right) \times R$.

Remarks on Proof. This is similar to the proof of Lemma 3.2. First one establishes a basic engulfing result which is similar to Lemma 3.1. Then (imitating the proof of Lemma 3.2) these basic engulfing moves are inductively "stacked" in order to produce our desired homeomorphism $\tilde{\theta}$ satisfying $d(f \tilde{\theta}, \theta f)<\varepsilon$. To achieve the second half of Theorem 3.3 all one has to do is use the $\nu$-control on the homotopies over $\left(X_{3}-\dot{X}_{1 / 3}\right) \times[-3,3]$ to further refine the above "stacking" so that we have $d(f \tilde{\theta}, \theta f)<\mu$ over $\left(X-\dot{X}_{2 / 3}\right) \times R$.

4. Wrapping up. In this section we will establish some results on wrapping up maps around $S^{1}$. Our main result is Theorem 4.3 , but as in $\$ 3$ we will treat the simpler global version first in Lemma 4.1 below. It is concerned with the problem of wrapping up $\delta$-fibrations around $S^{1}$. Theorem 4.2 is the analogue of Lemma 4.1 for wrapping up $\delta$-equivalences around $S^{1}$. For notation let $e: R \rightarrow S^{1}$ be the covering projection defined by $e(x)=\exp (\pi i x / 4)$, and let $X$ be a compact ANR with a specified metric.

LEMMA 4.1. For every $\varepsilon>0$ there exists $a \delta>0$ such that if $M$ is $a Q$-manifold and $f: M \rightarrow X \times R$ is a $\delta$-fibration over $X \times[-3,3]$, then there is a compact $Q$-manifold $\tilde{M}$, a map $\tilde{f}: \tilde{M} \rightarrow X \times S^{1}$ which is an $\varepsilon$-fibration, and an open embedding $\varphi: f^{-1}(X \times(-1,1)) \rightarrow \tilde{M}$ for which the following diagram commutes:

$$
\begin{array}{ccc}
\tilde{M} & \stackrel{\tilde{f}}{\rightarrow} & X \times S^{1} \\
\varphi \uparrow & & \uparrow \mathrm{id} \times e \\
f^{-1}(X \times(-1,1)) & \stackrel{f}{\rightarrow} & X \times(-1,1)
\end{array}
$$

Proof. We begin by constructing $\tilde{M}$. By Lemma 3.2 we can find a homotopy $h_{t}$ : $\mathrm{id}_{M} \simeq h_{1}$ which is supported on $f^{-1}(X \times[-2.5,2.5])$, such that $p_{1} f h_{t}$ is close to $p_{1} f$, and such that $h_{1}$ is a homeomorphism for which $p_{2} f h_{1} f^{-1}(X \times\{-t\})$ is close to $\{t+4\}$, for $1.8 \leqslant t \leqslant 2.2$. (Recall that $p_{1}$ denotes projection to $X$, and here we use $p_{2}$ to denote projection to $R$.) Let

$$
Y=h_{1} f^{-1}(X \times(-\infty,-2])-f^{-1}(X \times(-\infty,-2)),
$$

which is a compactum in $M$. Now define $\tilde{M}=Y / \sim$, where $\sim$ is the equivalence relation on $Y$ generated by $x \sim h_{1}(x)$, for all $x \in f^{-1}(X \times\{-2\})$. It is clear that $\tilde{M}$ is a compact $Q$-manifold.

We now define the map $\tilde{f}: \tilde{M} \rightarrow X \times S^{1}$. We can represent $S^{1}$ as $[-2,2] / \sim$, where $\sim$ is generated by $-2 \sim 2$. Thus $f^{-1}(X \times(-1,1))$ is naturally identified as an open subset of $\tilde{M}$ and $(-1,1)$ is naturally identified as an open subset of $S^{1}$. So we may regard $\varphi$ and $e \mid(-1,1)$ as inclusion maps in the commutative rectangle above. Now define a map $g: Y \rightarrow X \times[-2,2]$ as follows. The $R$-component of $g$, $p_{2} g$, sends $f^{-1}(X \times\{-2\})$ to -2 , it sends $h_{1} f^{-1}(X \times\{-2\})$ to +2 , and otherwise it is close to $p_{2} f \mid Y$. For the definition of $p_{1} g$ we will need the homotopy

$$
h_{t}^{-1}=h_{1-t} h_{1}^{-1}: i d \simeq h_{1}^{-1} \text {. }
$$


By the Estimated Homotopy Extension Theorem we can find a homotopy $e_{t}$ : $Y \rightarrow X$ for which $e_{0}=p_{1} f \mid Y, e_{t}=p_{1} f$ on $f^{-1}(X \times\{-2\}), e_{t}=p_{1} f h_{t}^{-1}$ on $h_{1} f^{-1}(X \times\{-2\})$, and $p_{1} e_{t}$ is close to $p_{1} f \mid Y$. Then define $p_{1} g=e_{1}$. Note that $g=f$ on $f^{-1}(X \times\{-2\})$ and $g=f h_{1}^{-1}$ on $h_{1} f^{-1}(X \times\{-2\})$. Thus $g$ factors through the appropriate equivalence relations to yield a map $\tilde{f}: \tilde{M} \rightarrow X \times S^{1}$ which makes the above rectangle commute.

There remains the problem of proving that $\tilde{f}$ is an $\varepsilon$-fibration. We clearly have a $\gamma$-homotopy $g \simeq f \mid Y$, where $\gamma$ is a small number. Thus by Proposition 2.2 it suffices to find a neighborhood of $X \times\{*\}$ (where * is the point of $S^{1}$ to which 2 and -2 have been identified) over which $\tilde{f}$ is an $\varepsilon_{1}$-fibration, for $\varepsilon_{1}$ small. Let

$$
U=h_{1} f^{-1}(X \times(-\infty,-1.8))-f^{-1}(X \times(-\infty, 1.8])
$$

and define $g^{\prime}: U \rightarrow X \times(1.8,2.2)$ by letting $p_{2} g^{\prime}=p_{2} g$ on $U \cap Y, p_{2} g^{\prime}=p_{2} g h_{1}^{-1}$ +4 on $U-Y, p_{1} g^{\prime}=p_{1} g$ on $U \cap Y$, and $p_{1} g^{\prime}=p_{1} g h_{1}^{-1}$ on $U-Y$. It is clear that we may regard $U$ as an open subset of $\tilde{M}$ and $X \times(1.8,2.2)$ as an open subset of $X \times S^{1}$ so that $\tilde{f} \mid U=g^{\prime}$. Thus if we can prove that $g^{\prime}$ is an $\varepsilon_{1}$-fibration over $X \times[1.9,2.1]$, then $\tilde{f}$ will be an $\varepsilon_{1}$-fibration over a neighborhood of $X \times\{*\}$ as desired.

By construction $p_{2} g^{\prime}$ is close to $p_{2} f \mid U$; thus there is a small homotopy $p_{2} g^{\prime} \simeq$ $p_{2} f \mid U$. We have a homotopy $p_{1} g^{\prime} \simeq u$, where $u: U \rightarrow X$ is defined by $u=p_{1} g$ on $U \cap Y$ and $u=p_{1} f h_{1}^{-1}$ on $U-Y$. This comes quickly from the homotopy $e_{1-t}$ : $p_{1} g \simeq p_{1} f \operatorname{rel} f^{-1}(X \times\{-2\})$. Now applying the homotopy $e_{1-t}$ again to $U \cap Y$ and extending it over $U$ we easily get a homotopy $u \simeq p_{1} f \mid U$. Putting all of this together we have a $\gamma$-homotopy $g^{\prime} \simeq f \mid U$, where $\gamma$ is small. Thus $g^{\prime}$ is an $\varepsilon_{1}$-fibration over $X \times[1.9,2.1]$ as desired.

We are now ready for our analogue of Lemma 4.1 for $\delta$-equivalences. In the following statement $p$ will always denote projection to $X \times R$ and $q$ will always denote projection to $X \times S^{1}$, where $X$ is again a compact ANR.

THEOREM 4.2. For every $\varepsilon>0$ there exists a $\delta>0$ such that if $M$ is $Q$-manifold, $F$ is a compact $A N R$, and $f: M \rightarrow X \times F \times R$ is a $p^{-1}(\delta)$-equivalence over $X \times F \times$ $[-3,3]$, then there is a compact $Q$-manifold $\tilde{M}, a q^{-1}(\varepsilon)$-equivalence $\tilde{f}: \tilde{M} \rightarrow X \times F$ $\times S^{1}$, and an open embedding $\varphi: f^{-1}(X \times F \times(-1,1)) \rightarrow \tilde{M}$ for which the following diagram commutes:

$$
\begin{array}{ccc}
\tilde{M} & \stackrel{\tilde{f}}{\rightarrow} & X \times F \times S^{1} \\
\varphi \uparrow & & \uparrow \mathrm{id} \times e \\
f^{-1}(X \times F \times(-1,1)) & \stackrel{f}{\rightarrow} & X \times F \times(-1,1)
\end{array}
$$

Remarks on Proof. This goes almost word-for-word like the proof of Lemma 4.1.

The following notation will be needed for Theorem 4.3. Let $X$ be an ANR with a specified metric and let $\varphi: X \rightarrow[0, \infty)$ be a proper map. For each $t \in[0, \infty)$ let $X_{t}=\varphi^{-1}([0, t])$, which is a compactum in $X$. 
TheORem 4.3. For every $\varepsilon>0$ there exists $a \delta>0$ such that if $M$ is a $Q$-manifold and $f: M \rightarrow X \times R$ is a $\delta$-fibration over $X_{3} \times[-3,3]$, then there exists a $Q$-manifold $\tilde{M}$, a map $\tilde{f}: \tilde{M} \rightarrow \dot{X}_{2.5} \times S^{1}$ which is an $\varepsilon$-fibration over $X_{2} \times S^{1}$, and an open embedding $\varphi: f^{-1}\left(\dot{X}_{1} \times(-1,1)\right) \rightarrow \tilde{M}$ so that the following diagram commutes:

$$
\begin{array}{ccc}
\tilde{M} & \stackrel{\tilde{f}}{\rightarrow} & \dot{X}_{2.5} \times S^{1} \\
\varphi \uparrow & & \uparrow \mathrm{id} \times e \\
f^{-1}\left(\dot{X}_{1} \times(-1,1)\right) & \stackrel{f}{\rightarrow} & \dot{\circ}_{1} \times(-1,1)
\end{array}
$$

Moreover, for every $\mu>0$ there exists a $\nu>0$ such that if $f$ is additionally given to be a $\nu$-fibration over $\left(X_{3}-\dot{X}_{1 / 3}\right) \times[-3,3]$, then the map $\tilde{f}: \tilde{M} \rightarrow \dot{X}_{2.5} \times S^{1}$ is additionally a $\mu$-fibration over $\left(X_{2}-\stackrel{\circ}{X}_{2 / 3}\right) \times S^{1}$.

Remarks on Proof. This is similar to the poof of Lemma 4.1. The only significant change that must be made is that Theorem 3.3 is used in lieu of Lemma 3.2 .

5. A handle lemma. The purpose of this section is to establish a result which is best described as a handle lemma for Theorem 1. Continuing the strategy of $\$ \S 3$ and 4 we first establish the global version (Lemma 5.1) and then quickly obtain the more general version (Theorem 5.2) as a corollary of the proof.

LeMmA 5.1. If $\varepsilon>0$ and $n>0$ are given, then there exists $a \delta>0$ so that for every $\mu>0, Q$-manifold $M$, and map $f: M \rightarrow R^{n}$ which is a $\delta$-fibration over $B_{3}^{n}$, there is a map $\tilde{f}: M \rightarrow R^{n}$ which is a $\mu$-fibration over $B_{1}^{n}$ and which is $\varepsilon$-homotopic to $f$ rel $M-f^{-1}\left(\check{B}_{3}^{n}\right)$.

Proof. We begin by introducing some notation. Let $e: R \rightarrow S^{1}$ be the covering projection defined in $\$ 4$ and let $e^{n}=e \times \cdots \times e: R^{n} \rightarrow T^{n}$ be the product covering projection. Thus $e^{n} \mid B_{3}^{n}: B_{3}^{n} \rightarrow T^{n}$ is an embedding. We regard $T^{n-1} \times R$ as a open subset of $\dot{B}_{3}^{n}$ so that $e^{n-1} \times$ id: $B_{2}^{n} \rightarrow B_{2}^{n}$ is the identity (see [11, §8]).

Now consider the restriction $f \mid: f^{-1}\left(T^{n-1} \times R\right) \rightarrow T^{n-1} \times R$. If $\delta$ is small enough, then $f \mid$ is a $\delta$-fibration over $T^{n-1} \times[-3,3]$. By Lemma 4.1 there is a compact $Q$-manifold $M^{\prime}$, a map $f^{\prime}: M^{\prime} \rightarrow T^{n}$ which is a $\delta^{\prime}$-fibration, and an open embedding $\varphi: f^{-1}\left(T^{n-1} \times(-2,2)\right) \rightarrow M^{\prime}$ so that the following diagram commutes:

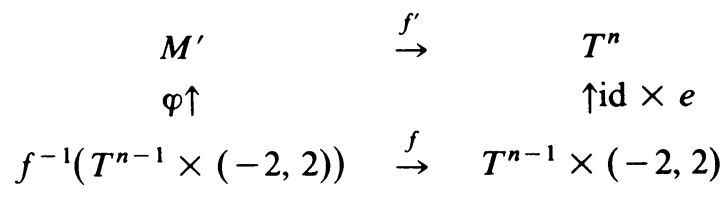

Recall that the size of $\delta^{\prime}$ depends on the size of $\delta$. In the remainder of the proof it will be convenient to regard $\varphi$ as an inclusion mapping.

We now unwrap all of this. By passing to covering spaces we obtain $f^{\prime \prime}$ : $M^{\prime \prime} \rightarrow R^{n}$ which makes the following pull-back diagram commute: 


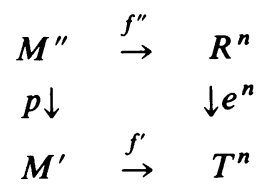

$M^{\prime \prime}$ is defined to be the subspace of $M^{\prime} \times R^{n}$ consisting of all pairs $(x, y)$ for which $f^{\prime}(x)=e^{n}(y)$. The maps $p$ and $f^{\prime \prime}$ are restrictions of projection maps.

Assertion. $M^{\prime \prime}$ is a $Q$-manifold and we can choose notation so that $f^{\prime \prime}=f$ over $\dot{B}_{2}^{n}$. Also $f^{\prime \prime}$ is a $\delta^{\prime \prime}$-fibration (globally), where the size of $\delta^{\prime \prime}$ depends on the size of $\delta^{\prime}$.

Proof. Since $e^{n}: R^{n} \rightarrow T^{n}$ is an immersion (i.e., locally an open embedding) and this is a pull-back diagram, it follows that the vertical map $p: M^{\prime \prime} \rightarrow M^{\prime}$ is also an immersion. Thus $M^{\prime \prime}$ is a $Q$-manifold. We chose $e^{n-1} \times$ id: $B_{2}^{n} \rightarrow B_{2}^{n}$ to be the identity, and from this it easily follows that $p \mid:\left(f^{\prime \prime}\right)^{-1}\left(\dot{B}_{2}^{n}\right) \rightarrow f^{-1}\left(\dot{B}_{2}^{n}\right)$ is a homeomorphism. Thus we can choose notation so that $f^{\prime \prime}=f$ over $\dot{B}_{2}^{n}$.

To see that $f^{\prime \prime}$ is a $\delta^{\prime \prime}$-fibration let $F: Z \times I \rightarrow R^{n}$ and $\tilde{F}_{0}: Z \rightarrow M^{\prime \prime}$ be maps for which $f^{\prime \prime} \tilde{F}_{0}=F_{0}$. Then $e^{n} F: Z \times I \rightarrow T^{n}$ and $p \tilde{F}_{0}: Z \rightarrow M^{\prime}$ are maps for which $f^{\prime} p \tilde{F}_{0}=e^{n} F_{0}$. Since $f^{\prime}$ is a $\delta^{\prime}$-fibration, there is a map $H: Z \times I \rightarrow M^{\prime}$ for which $H_{0}=p \tilde{F}_{0}$ and $f^{\prime} H$ is $\delta^{\prime}$-close to $e^{n} F$. Using the fact that $p: M^{\prime \prime} \rightarrow M^{\prime}$ is a fibration we can lift $H$ to $G: Z \times I \rightarrow M^{\prime \prime}$ so that $G_{0}=\tilde{F}_{0}$. Thus $f^{\prime \prime}$ is a $\delta^{\prime \prime}$-fibration. Alternately one could also invoke Proposition 2.2 to conclude that $f^{\prime \prime}$ is a $\delta^{\prime \prime}$-fibration.

We now modify the map $f^{\prime \prime}$. Choose $K>0$ large and let $\gamma: R^{n} \rightarrow R^{n}$ be a radially-defined homeomorphism which takes $B_{K}^{n}$ to $B_{3}^{n}$ and keeps $B_{2}^{n}$ pointwise fixed. Now define $\bar{f}=\gamma f^{\prime \prime}: M^{\prime \prime} \rightarrow R^{n}$. Clearly $\bar{f}=f$ over $\stackrel{B}{2}_{2}^{n}, \bar{f}$ is a $\delta^{\prime \prime \prime}$-fibration, and $\bar{f}$ is a $\mu$-fibration over $R^{n}-\dot{B}_{3}^{n}$, where the size of $\mu$ depends on the size of $1 / K$, and the size of $\delta^{\prime \prime \prime}$ depends on the size of $\delta^{\prime \prime}$.

The next step is to modify the map $\bar{f}$. Let $\theta: R^{n} \rightarrow R^{n}$ be a homeomorphism which is supported on $B_{8}^{n}$ and which is defined to be $\theta\left(x_{1}, x_{2}, \ldots, x_{n}\right)=\left(x_{1}+\right.$ $\left.5, x_{2}, \ldots, x_{n}\right)$ on $B_{2}^{n}$. This can be done so that $\theta$ only affects the first coordinate of $R^{n}$. Note that $\theta\left(B_{2}^{n}\right) \subset R^{n}-\dot{B}_{3}^{n}$. By the first part of Theorem 3.3 there is a homeomorphism $\tilde{\theta}: M^{\prime \prime} \rightarrow M^{\prime \prime}$ so that $\bar{f} \tilde{\theta}$ is $\varepsilon^{\prime \prime}$-close to $\theta \bar{f}$, where the size of $\varepsilon^{\prime \prime}$ depends on the size of $\delta^{\prime \prime \prime}$. Define $\hat{f}=\theta^{-1} \tilde{f} \tilde{\theta}: M^{\prime \prime} \rightarrow R^{n}$. This is a $\mu$-fibration over $B_{1.5}^{n}$. Moreover we observe that there is a homotopy of $\hat{f}$ to $\bar{f}$, where the size of this homotopy depends on the size of $\varepsilon^{\prime \prime}$.

Define $\tilde{f}: M \rightarrow R^{n}$ to be $\hat{f}$ over $B_{1.5}^{n}$, to be $f$ over $R^{n}-\dot{B}_{2}^{n}$, and over $B_{2}^{n}-\dot{B}_{1.5}^{n}$ we let $\tilde{f}$ be defined by the homotopy $\hat{f} \cong \bar{f}$ described above. It is clear that $\tilde{f}$ fulfills our requirements.

Before giving the statement of Theorem 5.2 we will have to introduce some more notation. Let $X$ be a compact ANR and let $c(X)$ denote the cone over $X$. More precisely, let $c(X)=X \times[0, \infty] / \sim$, where $\sim$ is the equivalence relation generated by $(x, 0) \sim\left(x^{\prime}, 0\right)$, for all $x, x^{\prime} \in X$. Similarly let $\dot{c}(X)=X \times[0, \infty) / \sim$ denote the open cone over $X$, and for any $t \in[0, \infty]$ let $c_{t}(X)=X \times[0, t] / \sim$ and $\dot{c}_{t}(X)=X \times[0, t) / \sim$. 
THEOREM 5.2. If $\varepsilon>0$ and $n \geqslant 0$ are given, then there exists a $\delta>0$ such that the following is true: for every $\mu>0$ there exists $a \nu>0$ so that if $M$ is a $Q$-manifold and $f: M \rightarrow \stackrel{c}{c}(X) \times R^{n}$ is a $\delta$-fibration over $c_{3}(X) \times B_{3}^{n}$, and a $\nu$-fibration over $\left[c_{3}(X)-\stackrel{\circ}{c}_{1 / 3}(X)\right] \times B_{3}^{n}$, then there is a map $\tilde{f:} M \rightarrow \stackrel{c}{c}(X) \times R^{n}$ which is a $\mu$-fibration over $c_{1}(X) \times B_{1}^{n}$ and which is $\varepsilon$-homotopic to $f$ rel $M-f^{-1}\left(\stackrel{\circ}{c}_{2 / 3}(X) \times \dot{B}_{3}^{n}\right)$.

Remarks on Proof. For $n \geqslant 1$ the proof is similar to the proof of Lemma 5.1. There are only two places in which any significant changes must be made.

The first occurs in the wrapping up procedure. In the more general case that we are dealing with, Theorem 4.3 must be used in lieu of Lemma 4.1 in order to accommodate the $\dot{c}(X)$-factor. The second occurs in the construction of the map $\bar{f}=\gamma f^{\prime}$, where $\gamma$ is a "radial squeeze" towards the origin. In this more general case, $\gamma: \stackrel{\circ}{c}(X) \times R^{n} \rightarrow \stackrel{\circ}{c}(X) \times R^{n}$ is a homeomorphism which is a composition $\gamma=\gamma_{2} \gamma_{1}$, where $\gamma_{1}$ gives a squeeze to $R^{n}$ towards the origin and $\gamma_{2}$ gives a squeeze to $\dot{c}(X)$ towards the vertex. This device of squeezing towards the vertex is amply illustrated in the case $n=0$ below.

We now treat the case $n=0$, which is not covered in the proof of Lemma 5.1. We are given $\varepsilon>0, \mu>0$ and a proper map $\tilde{f}: M \rightarrow \stackrel{c}{c}(X)$ which is a $\delta$-fibration over $c_{3}(X)$ and a $\nu$-fibration over $c_{3}(X)-\dot{c}_{1 / 3}(X)$. Choose $t$ close to 0 and let $\theta$ : $\grave{c}(X) \rightarrow \grave{c}(X)$ be a homeomorphism which is supported on $c_{2 / 3}(X)-\dot{c}_{t / 2}(X)$ and which takes $c_{t}(X)$ to $c_{1 / 2}(X)$. This can be done so that $\theta$ affects only the $[0, \infty)$-coordinates in $\stackrel{c}{c}(X)$. By Lemma 3.1 there is a homeomorphism $\tilde{\theta}: M \rightarrow M$ for which $f \tilde{\theta}^{-1}$ is $\tilde{\delta}$-close to $\theta^{-1} f$, where the size of $\tilde{\delta}$ is dependent on the size of $\delta$. Then $f^{\prime}=\theta^{-1} f \tilde{\theta}: \quad M \rightarrow \grave{c}(X)$ is a $\tilde{\mu}$-fibration over $c_{3}(X)-\check{c}_{t}(X)$ and which is $\varepsilon / 2$-homotopic to $f$ rel $M-f^{-1}\left(\stackrel{c}{c}_{2 / 3}(X)\right)$, where the $\varepsilon / 2$-homotopy comes from

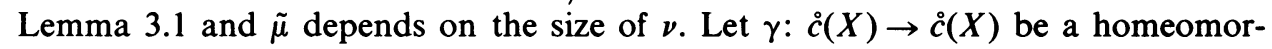
phism which is supported on $c_{2 / 3}(X)$ and which squeezes $c_{t}(X)$ close to the vertex. It is then clear that $\tilde{f}=\gamma f^{\prime}$ fulfills our requirements.

6. Proof of Theorem 1 and its corollary. We have divided the proof of Theorem 1 into three cases which are treated in order of increasing generality. They are: $B$ is a polyhedron, $B$ is a $Q$-manifold, and $B$ is an ANR.

I. $B$ is a polyhedron. We are given a $\beta$-fibration $f: M \rightarrow B$. We will construct sequences $\left\{f_{i}\right\}$ and $\left\{\beta_{i}\right\}$ such that $f_{i}: M \rightarrow B$ is a $\beta_{i}$-fibration, $\lim _{i \rightarrow \infty} \operatorname{mesh}\left(\beta_{i}\right)=$ 0 , where

$$
\operatorname{mesh}\left(\beta_{i}\right)=\operatorname{lub}\left\{\operatorname{diam}(U) \mid U \in \beta_{i}\right\}
$$

$f_{i}$ is close to $f$, and $p=\lim _{i \rightarrow \infty} f_{i}: M \rightarrow B$ is a proper map. Certainly $p$ must be close to $f$, and to see that $p$ is an approximate fibration it suffices (by Proposition 2.2) to show that for every compactum $C \subset B$ and open cover $\gamma$ of $B, p$ is a $\gamma$-fibration over $C$. So suppose that we have maps $F: Z \times I \rightarrow C$ and $\tilde{F}_{0}$ : $Z \rightarrow p^{-1}(C)$ such that $p \tilde{F}_{0}=F_{0}$. If $i$ is large, then there is a small homotopy of $p \mid p^{-1}(C)$ to $f_{i} \mid p^{-1}(C)$. Since $p \tilde{F}_{0}=F_{0}$ this gives us a small homotopy of $F_{0}$ to $f_{i} \tilde{F}_{0}$. By the Estimated Homotopy Extension Theorem we can get a small homotopy $F \simeq F^{\prime}$ so that $F_{0}^{\prime}=f_{i} \tilde{F}_{0}$. Since $f_{i}$ has the $\beta_{i}$-lifting property we have a map $G$ : 
$Z \times I \rightarrow M$ so that $G_{0}=\tilde{F}_{0}$ and $f_{i} G$ is $\beta_{i}$-close to $F^{\prime}$. Then $p G$ is close to $F$ as desired.

All that remains to be done for this case is the verification of the existence of the sequences $\left\{f_{i}\right\}$ and $\left\{\beta_{i}\right\}$. They are inductively chosen by the following

Assertion. For every open cover $\alpha$ of $B$ there exists an open cover $\beta$ of $B$ so that the following is true: if $M$ is a $Q$-manifold and $f: M \rightarrow B$ is a $\beta$-fibration, then for every open cover $\gamma$ of $B$ there exists a $\gamma$-fibration $f^{\prime}: M \rightarrow B$ which is $\alpha$-close to $f$.

Proof. We will need a "handle decomposition" of $B$. Assume that $B$ has a fixed triangulation and for each barycenter $b \in B$ let $C_{b}$ be the closed star of $b$ in the second barycentric subdivision of $B$. Recalling the cone notation of $\$ 5$ we can regard each $C_{b}$ as $c_{1}\left(X_{b}\right) \times B_{1}^{n}$, where $X_{b}$ is a compact polyhedron and $B_{1}^{n}$ is a neighborhood of $b$ in the simplex of which $b$ is the barycenter. By enlarging $C_{b}$ slightly we obtain an open set in $B$ of the form $\stackrel{c}{c}\left(X_{b}\right) \times R^{n}$ so that $C_{b}$ is identified with $c_{1}\left(X_{b}\right) \times B_{1}^{n} \subset \dot{c}\left(X_{b}\right) \times R^{n}$. (If $b$ is the barycenter of a principal simplex, then we let $\stackrel{c}{c}\left(X_{b}\right)=\{b\}$ for convenience.) If the choices are correctly made, then for any fixed integer $k$ the sets $\stackrel{c}{c}\left(X_{b}\right) \times R^{k}$ are pairwise-disjoint.

If $B$ is compact then the procedure is standard. Let $\operatorname{dim} B=n$ and consider the collection $\left\{\stackrel{c}{c}\left(X_{b} \times R^{n}\right) \mid b\right.$ is the barycenter of some $n$-simplex $\}$. The open sets of this collection are pairwise-disjoint, so the handle lemma of $\$ 5$ can be applied to each of them (independently). Since $c\left(X_{b}\right)=\{b\}$ we only need Lemma 5.1. If $\beta$ is sufficiently fine, then Lemma 5.1 yields a $\beta_{1}$-fibration $f_{1}: M \rightarrow B$ which is close to $f$ and which is a $\gamma_{1}$-fibration over a neighborhood of $S_{n}=\cup\left\{C_{b} \mid b\right.$ is the barycenter of some $n$-simplex $\}$, where $\operatorname{mesh}\left(\beta_{1}\right)$ depends on $\operatorname{mesh}(\beta)$ and $\operatorname{mesh}\left(\gamma_{1}\right)$ can be chosen as small as we want. This completes the first step of the construction.

The next step is to treat similarly the open sets $c\left(X_{b}\right) \times R^{n-1}$, where $b$ is the barycenter of some $(n-1)$-simplex. In this case we use the full strength of the handle lemma (i.e., Theorem 5.2) to find similarly a $\beta_{2}$-fibration $f_{2}: M \rightarrow B$ which is close to $f_{1}$ and which a $\gamma_{2}$-fibration over a neighborhood of $S_{n} \cup S_{n-1}$, where $S_{n-1}=\cup\left\{C_{b} \mid b\right.$ is the barycenter of some $(n-1)$-simplex $\}$, where $\operatorname{mesh}\left(\beta_{2}\right)$ depends on $\operatorname{mesh}\left(\beta_{1}\right)$, and where $\operatorname{mesh}\left(\gamma_{2}\right)$ depends on $\operatorname{mesh}\left(\gamma_{1}\right)$. This completes the second step of the construction. It is now clear that one can continue in this manner until all of the handles in $B$ are exhausted.

If $B$ is not compact, then one writes $B$ as a union of compact subpolyhedra, $B=\cup B_{i}$, so that $B_{i} \cap B_{j}=\varnothing$ for $|i-j| \geqslant 2$. Then the above procedure for the compact case is applied to the $B_{2 i-1}$ (independently) to produce a $\beta_{0}$-fibration $f_{0}$ : $M \rightarrow B$ which is close to $f$ and which is a $\gamma_{0}$-fibration over a neighborhood of $\cup B_{2 i-1}$, where on any $B_{2 i-1} \operatorname{mesh}\left(\beta_{0}\right)$ depends on $\operatorname{mesh}(\beta)$ and $\operatorname{mesh}\left(\gamma_{0}\right) \operatorname{can}$ be made as small as we want. Again applying the procedure for the compact case to the $B_{2 i}$ (independently) we produce our desired $\gamma$-fibration $f^{\prime}: M \rightarrow B$ which is $\alpha$-close to $f$.

II. $B$ is a $Q$-manifold. Let $f: M \rightarrow B$ be a $\beta$-fibration, where $B$ is a $Q$-manifold. According to the proof of the polyhedral case, it suffices to prove that for every open cover $\gamma$ of $B, f$ is $\alpha$-close to a $\gamma$-fibration. Choose a factorization, $B=B_{1} \times$ $Q$, where $B_{1}$ is a polyhedron and so that each $\{x\} \times Q$ is small with respect to the open cover $\alpha$. Let $p=$ proj: $B \rightarrow B_{1}$. 
Assertion 1.pf: $M \rightarrow B_{1}$ is a $\beta_{1}$-fibration, where $\beta_{1}=p(\beta)=\{p(U) \mid U \in \beta\}$.

Proof. Let $F: Z \times I \rightarrow B_{1}$ and $\tilde{F}_{0}: Z \rightarrow M$ be maps such that $p f \tilde{F}_{0}=F_{0}$. Define $H: Z \times I \rightarrow B_{1} \times Q$ by $H(z, t)=\left(F(z, t), p_{Q} f \tilde{F}_{0}(z)\right)$, where $p_{Q}=$ proj: $B \rightarrow Q$. Note that $H_{0}=f \tilde{F}_{0}$ and $p H=F$. Since $f$ is a $\beta$-fibration, there is a map $\tilde{H}$ : $Z \times I \rightarrow M$ such that $\tilde{H}_{0}=\tilde{F}_{0}$ and such that $f \tilde{H}$ is $\beta$-close to $H$. Then $p f \tilde{H}$ is $\beta_{1}$-close to $p H=F$ and we are done.

If $\gamma_{1}$ is any open cover of $B_{1}$, then we can use the polyhedral case to find a $\gamma_{1}$-fibration $\bar{f}: M \rightarrow B_{1}$ which is close to $p f$. This cover $\gamma_{1}$ can be chosen as fine as we want provided that a correspondingly fine choice of $\beta$ is made.

Assertion 2. If $\gamma_{1}$ is sufficiently fine, then $\bar{f} \times \mathrm{id}: M \times Q \rightarrow B_{1} \times Q$ is a $\gamma$-fibration.

Proof. Let $F: Z \times I \rightarrow B_{1} \times Q$ and $\tilde{F}_{0}: Z \rightarrow M \times Q$ be maps such that $F_{0}=\left(\bar{f} \times\right.$ id) $\tilde{F}_{0}$. If $p_{M}=$ proj: $M \times Q \rightarrow M$, then we clearly have a map $\hat{G}$ : $Z \times I \rightarrow M$ such that $\bar{f} \hat{G}$ is $\gamma_{1}$-close to $p F$ and such that $\hat{G}_{0}=p_{M} \tilde{F}_{0}$. Define $G$ : $Z \times I \rightarrow M \times Q$ by $G(z, t)=\left(\hat{G}(z, t), p_{Q} F(z, t)\right)$. Then $G_{0}=\tilde{F}_{0}$ and $(\bar{f} \times$ id $) G$ is $\gamma$-close to $F$.

Now let $u: M \times Q \rightarrow M$ be a homeomorphism which is close to projection. Then $f^{\prime}=(\bar{f} \times$ id $) u^{-1}: M \rightarrow B$ is a $\gamma$-fibration which is $\alpha$-close to $f$ because of the size of the sets $\{x\} \times Q$. This completes the $Q$-manifold case.

III. $B$ is an $A N R$. Again it suffices to show that the $\beta$-fibration $f: M \rightarrow B$ is $\alpha$-close to a $\gamma$-fibration. By Assertion 2 above it follows that $f \times$ id: $M \times Q \rightarrow B$ $\times Q$ is a $\beta_{1}$-fibration, where $\beta_{1}$ can be made as fine as we want provided that we make a correspondingly fine choice of $\beta$. Since $B \times Q$ is a $Q$-manifold [6, p. 106], we can use the $Q$-manifold case above to find a $\gamma_{1}$-fibration $\bar{f}: M \times Q \rightarrow B \times Q$ which is close to $f \times$ id. We can choose $\gamma_{1}$ as fine as we want. If $u: M \times Q \rightarrow M$ is a homeomorphism close to projection, then

$$
f^{\prime}: M \stackrel{u^{-1}}{\rightarrow} M \times Q \stackrel{\bar{f}}{\rightarrow} B \times Q \stackrel{\text { proj }}{\rightarrow} B
$$

is a $\gamma$-fibration which is $\alpha$-close to $f$ (see Assertion 1 above). This completes the proof of the ANR case and therefore the proof of Theorem 1.

Proof of Corollary. Since $K$ is finitely dominated, $K \times S^{1}$ has the homotopy type of a compact polyhedron [15]. Thus there is a compact $Q$-manifold $M_{1}$ and a homotopy equivalence $f_{1}: M_{1} \rightarrow K \times S^{1}$. Let $\nu: S^{1} \rightarrow S^{1}$ be the map $\nu(z)=z^{n}$ (the standard $n$-fold covering map), and form the pull-back diagram:

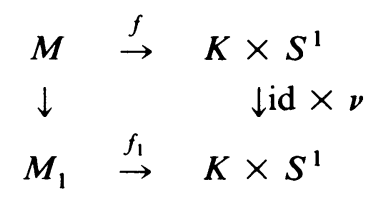

Then $M$ is a compact $Q$-manifold and $f: M \rightarrow K \times S^{1}$ is still a homotopy equivalence. More importantly, proj $\circ f: M \rightarrow S^{1}$ is an $\varepsilon$-fibration, where $\varepsilon=\varepsilon(n)$ is small if $n$ is large. This is an easy exercise using the basic properties of covering spaces. By Theorem 1 we have proj $\circ f$ homotopic to an approximate fibration $p$ : $M \rightarrow S^{1}$. The homotopy fiber of $p$ is homotopy equivalent to the homotopy fiber of 
proj: $K \times S^{1} \rightarrow S^{1}$ because the homotopy fiber is an invariant of homotopy type. But the homotopy fiber of proj: $K \times S^{1} \rightarrow S^{1}$ is $K^{4}$

7. Some preliminaries for Theorem 2. The purpose of this section is twofold: first we recall some well-known facts from simple homotopy theory and algebraic $K$-theory, and then we put all of this material together to establish some lemmas which will be needed for the proof of Theorem 2 .

We begin by recalling the geometric interpretation of the Whitehead group of a compact polyhedron which is given in $[8, \S 6]$. For any compact polyhedron $L$ the Whitehead group of $L, \mathrm{Wh}(L)$, can be realized geometrically as the set of all equivalence classes $[K, L]$, where $(K, L)$ is a compact polyhedral pair for which $K$ strong deformation retracts to $L$. Two of these equivalence classes, $[K, L]$ and $\left[K^{\prime}, L\right]$, are equal provided that there is a simple homotopy equivalence $g: K \rightarrow K^{\prime}$ for which the following homotopy commutes:

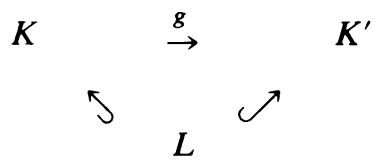

Each equivalence class represents the torsion, $\tau(f)$, of a strong deformation retraction $f: K \rightarrow L$.

Now let $q: L \rightarrow B$ be a map to some compact parameter space. We say that the torsion $[K, L]$ is a $q^{-1}(\varepsilon)$-torsion provided that we can choose a strong deformation retraction $f: K \rightarrow L$ for which $f \simeq$ id rel $L$ via a $q^{-1}(\varepsilon)$-homotopy. We call $f$ a $q^{-1}(\varepsilon)$-strong deformation retraction. It is obvious that if $[K, L]$ and $\left[K^{\prime}, L\right]$ are $q^{-1}(\varepsilon)$-torsions, then so is the sum $[K, L]+\left[K^{\prime}, L\right]=\left[K \cup_{L} K^{\prime}, L\right]$. On p. 21 of [8] there is given a geometric construction of the inverse, $-[K, L]$, of a torsion $[K, L]$. It follows from this that for every $\varepsilon>0$ there exists a $\delta>0$ so that if $[K, L]$ is a $q^{-1}(\delta)$-torsion, then $-[K, L]$ is a $q^{-1}(\varepsilon)$-torsion. In fact we may choose $\delta=\varepsilon / 3$, and to prove this all we need is the following result: If $f: K \rightarrow L$ is $a$ $q^{-1}(\delta)$-strong deformation retraction and $c: M(f) \rightarrow L$ is the collapse of the mapping cylinder to its base, then there is a $(q c)^{-1}(3 \delta)$-strong deformation retraction of $M(f)$ to the top $K$. This is an easy exercise using the Estimated Homotopy Extension Theorem and the construction of $[17$, p. 31] for converting a weak deformation retraction to a strong deformation retraction.

Our main object of concern in this section is the fundamental theorem of algebraic $K$-theory [2, Chapter XII]. In what follows $q$ will denote projection to $S^{1}$, the set of complex numbers of modulus 1 . For any compact polyhedron $L$ there is a functorial direct sum decomposition,

$$
\mathrm{Wh}\left(S^{1} \times L\right)=\mathrm{Wh}(L) \oplus \tilde{K}_{0}(L) \oplus \operatorname{Nil}(L),
$$

where $\operatorname{Nil}(L)$ is a term which will be of no use to us. The canonical injection $\mathrm{Wh}(L) \rightarrow \mathrm{Wh}\left(S^{1} \times L\right)$ is realized geometrically by the rule $[K, L] \mapsto\left[\left(S^{1} \times L\right) \cup\right.$ $\left.(\{1\} \times K), S^{1} \times L\right]$. It follows that the torsions in $\mathrm{Wh}\left(S^{1} \times L\right)$ which lie in

\footnotetext{
[13].

${ }^{4}$ This is clarified by the fact that the homotopy fiber of $p$ is shape equivalent to $p^{-1}(z)$, for all $z \in S^{1}$
} 
$\mathrm{Wh}(L)$ are all $q^{-1}(\varepsilon)$-torsions, for any $\varepsilon>0$. The canonical retraction $\mathrm{Wh}\left(S^{1} \times L\right)$ $\rightarrow \mathrm{Wh}(L)$ is realized geometrically by the rule $\left[K, S^{1} \times L\right] \mapsto\left[K \cup\left(D^{2} \times L\right),\{1\}\right.$ $\times L]$, where $D^{2}$ is the disc bounding $S^{1}$ and $K \cap\left(D^{2} \times L\right)=S^{1} \times L$.

The canonical injection $\tilde{K}_{0}(L) \rightarrow \mathrm{Wh}\left(S^{1} \times L\right)$ is not quite as easy to realize geometrically. Assuming that $L$ is connected let $[P] \in \tilde{K}_{0}(L)$, where $P$ is a finitely generated projective $Z\left[\pi_{1}(L)\right]$-module. (If $L$ is not connected, then we can direct sum over the components.) Choose another f.g. projective $Z\left[\pi_{1}(L)\right]$-module $P^{\prime}$ for which $P \oplus P^{\prime}$ is free of dimension $k$. Let $X=L \vee\left(\bigvee_{i=1}^{k} S_{i}^{2}\right)$, which is the space formed by wedging a bouquet of $k$ 2-spheres with $L$, and let $Y=\left(S^{1} \times L\right) \cup(\{1\}$ $\times X)$. The canonical injection $\tilde{K}_{0}(L) \rightarrow \mathrm{Wh}\left(S^{1} \times L\right)$ will send $[P]$ to $\left[\tilde{Y}, S^{1} \times L\right]$, where $\tilde{Y}$ is the space formed by attaching $k$ 3-cells to $Y$ via attaching maps $\psi_{i}$ which are described as follows. Let $* \in Y$ be the wedge point and for each $i$ let $\varphi_{i}$ : $\left(S^{2}, b_{0}\right) \rightarrow\left(S_{i}^{2}, *\right)$ be a homeomorphism. The homotopy classes $\left[\varphi_{i}\right]$ are the free generators of the $Z\left[\pi_{1}(L)\right]$-module $\pi_{2}(X, L)$. Also there is an isomorphism of $Z\left[\pi_{1}(L)\right]$-modules, $\pi_{2}(X, L) \approx P \oplus P^{\prime}$. The retraction $P \oplus P^{\prime} \rightarrow P$ induces a $Z\left[\pi_{1}(L)\right]$-module homomorphism $\alpha: \pi_{2}(X, L) \rightarrow \pi_{2}(X, L)$. Now identify each $\left[\varphi_{i}\right]$ with its natural image in $\pi_{2}\left(Y, S^{1} \times L\right)$ and let $\psi_{i}:\left(S^{2}, b_{0}\right) \rightarrow(Y, *)$ represent the homotopy class $\alpha\left[\varphi_{i}\right]+t(1-\alpha)\left[\varphi_{i}\right]$, where multiplication by $t$ indicates the action of the generator of $\pi_{1}\left(S^{1}\right) \subset \pi_{1}(Y)$ on $\pi_{2}\left(Y, S^{1} \times L\right)$. Then $\tilde{Y}$ is formed by attaching 3-cells to $Y$ via the attaching maps $\psi_{i}, 1 \leqslant i \leqslant k$. This completes the description of the injection $\tilde{K}_{0}(L) \rightarrow \mathrm{Wh}\left(S^{1} \times L\right)$.

The above construction for the injection $\tilde{K}_{0}(L) \rightarrow \mathrm{Wh}\left(S^{1} \times L\right)$ can be iterated by wedging on the bouquets $\bigvee_{i=1}^{k} S_{i}^{2}$ any finite number of times around $S^{1} \times L$, and then attaching corresponding blocks of $k$ 3-cells to kill the generators of $\pi_{2}\left(Y, S^{1} \times L\right)$. This "stacking" procedure yields the same homomorphism of $\tilde{K}_{0}(L)$ into $\mathrm{Wh}\left(S^{1} \times L\right)$, and it is also the same one that is obtained upon passage to standard finite covers of $S^{1}$ by taking pull-backs as in the proof of the Corollary of Theorem 1. It then follows that each torsion in $\mathrm{Wh}\left(S^{1} \times L\right)$ which lies in $\tilde{K}_{0}(L)$ is a $q^{-1}(\varepsilon)$-torsion for any $\varepsilon>0$. We summarize the situation as follows.

Lemma 7.1. Any torsion in $\mathrm{Wh}\left(S^{1} \times L\right)$ which lies in $\mathrm{Wh}(L) \oplus \tilde{K}_{0}(L)$ is a $q^{-1}(\varepsilon)$-torsion, for any $\varepsilon>0$.

Here is a result which says that Lemma 7.1 is the best possible result.

LEMMA 7.2. There exists an $\varepsilon>0$ so that if $K, L$ are compact polyhedra and $[K, L]$ is a $q^{-1}(\varepsilon)$-torsion in $\mathrm{Wh}\left(S^{1} \times L\right)$, then $[K, L] \in \mathrm{Wh}(L) \oplus \tilde{K}_{0}(L)$.

Proof. If $\tau_{1}$ is the component of $[K, L]$ in $\tilde{K}_{0}(L) \subset \mathrm{Wh}\left(S^{1} \times L\right)$, then by the above remarks the torsion $-\tau_{1}$ is also a $q^{-1}(\varepsilon)$-torsion. Thus we can find a compact polyhedral pair $\left(J, S^{1} \times L\right)$ so that $\left[J, S^{1} \times L\right]=[K, L]-\tau_{1}$ is a $q^{-1}(\varepsilon)$-torsion. So all we have to do is prove that $\left[J, S^{1} \times L\right] \in \mathrm{Wh}(L) \subset$ $\mathrm{Wh}\left(S^{1} \times L\right)$.

In what follows it will be convenient to assume that $J$ is a compact $Q$-manifold. The Classification Theorem of [6, Chapter 12] assures us that there is no loss of generality in making such an assumption. Let $e: R \rightarrow S^{1}$ be the covering projection 
used in $\$ 4$ and form the pull-back diagram:

$$
\begin{array}{ccc}
\tilde{J} & \stackrel{\tilde{f}}{\rightarrow} & R \times L \\
s \downarrow & & \downarrow e \times \mathrm{id} \\
J & \stackrel{f}{\rightarrow} & S^{1} \times L
\end{array}
$$

where $f: J \rightarrow S^{1} \times L$ is a $q^{-1}(\varepsilon)$-strong deformation retraction. Then $\tilde{f}$ is a $p^{-1}(\delta)$-equivalence, where $p=$ projection to $R$ and $\delta$ is small. There is an obstruction in $\tilde{K}_{0}(L)$ to splitting $\tilde{J}$, i.e., to finding a compact bicollared $Q$-manifold $M \subset \tilde{J}$ so that $M \hookrightarrow \tilde{J}$ is a homotopy equivalence [6, Chapter IX]. By Proposition 4.7 of [16] this obstruction in $\tilde{K}_{0}(L)$ is the $\tilde{K}_{0}(L)$-component of $\tau(f)=[J, L]$, which is 0 . Thus the splitting manifold $M \subset \tilde{J}$ exists. By Lemma 3.2 we can assume that $M \subset \tilde{f}^{-1}((-1,1) \times L)$, and so $s: M \rightarrow J$ is an embedding. This enables us to write $J=A \cup B$ and homotope $f$ to $f^{\prime}: J \rightarrow S^{1} \times L$ so that

(1) $A$ and $B$ are compact $Q$-manifolds such that $A \cap B=M_{1} \cup M_{2}$, where $M_{1}, M_{2}$ are $Q$-manifolds obtained in a manner similar to $s(M)$ and $M_{1} \cap M_{2}=\varnothing$;

(2) $M_{1} \hookrightarrow A$ and $M_{1} \hookrightarrow B$ are homotopy equivalences;

(3) $f^{\prime}(A)=S_{A}^{1} \times L, f^{\prime}(B)=S_{B}^{1} \times L$, and $f^{\prime}\left(M_{1} \cup M_{2}\right)=\left\{a_{1}, a_{2}\right\} \times L$, where $S_{A}^{1}$ and $S_{B}^{2}$ are arcs in $S^{1}$ which meet in $\left\{a_{1}, a_{2}\right\}$;

(4) $f^{\prime}\left|A: A \rightarrow S_{A}^{1} \times L, f^{\prime}\right| B: B \rightarrow S_{B}^{1} \times L, f^{\prime} \mid M_{1}: M_{1} \rightarrow\left\{a_{1}\right\} \times L$, and $f^{\prime} \mid M_{2}$ : $M_{2} \rightarrow\left\{a_{2}\right\} \times L$ are all homotopy equivalences.

By the Sum Theorem for Whitehead torsion [8, p. 76], we conclude that $\tau\left(f^{\prime}\right) \in \mathrm{Wh}(L)$ as desired.

The remainder of this section is concerned with generalizations of Lemma 7.1 and 7.2. We may write the $n$-torus as $T^{n}=S_{1}^{1} \times \cdots \times S_{n}^{1}$, and for each $i$ we define $T_{i}^{n-1}=S_{1}^{1} \times \cdots \times \hat{S}_{i}^{1} \times \cdots \times S_{n}^{1}$. Then for each $i$ we get a factorization $T^{n}=S_{i}^{1} \times T_{i}^{n-1}$, which gives us a direct sum decomposition

$$
\mathrm{Wh}\left(T^{n} \times L\right)=\mathrm{Wh}\left(T_{i}^{n-1} \times L\right) \oplus \tilde{K}_{0}\left(T_{i}^{n-1} \times L\right) \oplus \operatorname{Nil}\left(T_{i}^{n-1} \times L\right) .
$$

We define $\tilde{K}_{1-n}(L)=\cap_{i=1}^{n} \tilde{K}_{0}\left(T_{i}^{n-1} \times L\right)$, which then gives us the direct sum decomposition mentioned in $\S 1$ (see $[1$, p. 220]):

$$
\mathrm{Wh}\left(T^{n} \times L\right)=\mathrm{Wh}(L) \oplus \sum_{i=1}^{n}\left(\begin{array}{c}
n \\
i
\end{array}\right) \tilde{K}_{1-i}(L) \oplus \text { Nil terms. }
$$

In what follows $q$ will denote projection to $T^{n}$.

LEMMA 7.3. Any torsion in $\mathrm{Wh}\left(T^{n} \times L\right)$ which lies in $\mathrm{Wh}(L) \oplus \sum_{i=1}^{n}\left(\begin{array}{l}n \\ i\end{array}\right) \tilde{K}_{1-i}(L)$ is a $q^{-1}(\varepsilon)$-torsion, for any $\varepsilon>0$.

Proof. The proof is by induction on $n$, with the case $n=1$ having been done in Lemma 7.1. Looking at the inductive step we now assume the result to be true for some $n$, and then prove it to be true for $n+1$. For $n+1$ the direct sum decompositions mentioned above become

$$
\mathrm{Wh}\left(T^{n+1} \times L\right)=\mathrm{Wh}\left(T_{i}^{n} \times L\right) \oplus \tilde{K}_{0}\left(T_{i}^{n} \times L\right) \oplus \operatorname{Nil}\left(T_{i}^{n} \times L\right)
$$


and

$$
\mathrm{Wh}\left(T^{n+1} \times L\right)=\mathrm{Wh}(L) \oplus \sum_{i=1}^{n+1}\left(\begin{array}{c}
n+1 \\
i
\end{array}\right) \tilde{K}_{1-i}(L) \oplus \text { Nil terms. }
$$

If $q_{i}: T_{i}^{n} \times L \rightarrow T_{i}^{n}$ is projection, then our inductive hypothesis says that each torsion in the subgroup $G_{i}=\mathrm{Wh}(L) \oplus \sum_{i=1}^{n}\left(\begin{array}{c}n \\ i\end{array}\right) \tilde{K}_{1-i}(L)$ of $\mathrm{Wh}\left(T_{i}^{n} \times L\right)$ is a $q_{i}^{-1}(\varepsilon)$-torsion, for any $\varepsilon>0$. From the manner in which the injection

$$
\mathrm{Wh}\left(T_{i}^{n} \times L\right) \rightarrow \mathrm{Wh}\left(T^{n+1} \times L\right)
$$

was described at the begining of this section, we conclude that each torsion in the subgroup $G_{i}$ of $\mathrm{Wh}\left(T^{n+1} \times L\right)$ is a $q^{-1}(\varepsilon)$-torsion, for any $\varepsilon>0$, where $q$ : $T^{n+1} \times L \rightarrow T^{n+1}$ is projection. Each torsion in $\sum_{i=1}^{n}\left({ }^{n+1}{ }_{i}\right) \tilde{K}_{1-i}(L)$ lies in one of the $G_{i}$ 's; thus each torsion in $\mathrm{Wh}(L) \oplus \sum_{i=1}^{n}\left(\begin{array}{c}n+1 \\ i\end{array}\right) \tilde{K}_{1-i}(L)$ is a $q^{-1}(\varepsilon)$-torsion, for any $\varepsilon>0$.

The only torsions left to examine are those in

$$
\tilde{K}_{-n}(L)=\bigcap_{i=1}^{n+1} \tilde{K}_{0}\left(T_{i}^{n} \times L\right) .
$$

In the paragraph preceding Lemma 7.1 we have already observed that the torsions in $\tilde{K}_{0}\left(T_{i}^{n} \times L\right)$ are invariant under passage to standard finite covers of the $S_{i}^{1}$-factor of $T^{n+1}=S_{i}{ }^{1} \times T_{i}^{n}$. Thus the torsions in $\tilde{K}_{n}(L)$ are invariant under passage to standard finite covers $T^{n+1} \rightarrow T^{n+1}$. Just as in the proof of the Corollary of Theorem 1 , we conclude that any torsion in $\tilde{K}_{-n}(L)$ is a $q^{-1}(\varepsilon)$-torsion, for any $\varepsilon>0$.

LEMMA 7.4. There exists an $\varepsilon>0$ so that if $K, L$ are compact polyhedra and $[K, L]$ is a $q^{-1}(\varepsilon)$-torsion in $\mathrm{Wh}\left(T^{n} \times L\right)$, then

$$
[K, L] \in \mathrm{Wh}(L) \oplus \sum_{i=1}^{n}\left(\begin{array}{c}
n \\
i
\end{array}\right) \tilde{K}_{1-i}(L) .
$$

Proof. Again we induct on $n$, with the case $n=1$ having been done in Lemma 7.2. Assume the result to be true for some $n$ and now consider the case $n+1$. Using the direct sum decomposition,

$$
\mathrm{Wh}\left(T^{n+1} \times L\right)=\mathrm{Wh}\left(T_{i}^{n} \times L\right) \oplus \tilde{K}_{0}\left(T_{i}^{n} \times L\right) \oplus \operatorname{Nil}\left(T_{i}^{n} \times L\right)
$$

we conclude from Lemma 7.2 that the $q^{-1}(\varepsilon)$-torsion $[K, L]$ lies in $\mathrm{Wh}\left(T_{i}^{n} \times L\right) \oplus$ $\tilde{K}_{0}\left(T_{i}^{n} \times L\right)$. The canonical retraction of $\mathrm{Wh}\left(T^{n+1} \times L\right)$ to $\mathrm{Wh}\left(T_{i}^{n} \times L\right)$, described at the beginning of this section, implies that the component of $[K, L]$ in $\mathrm{Wh}\left(T_{i}^{n} \times L\right)$ is a $q_{i}^{-1}\left(\varepsilon_{i}\right)$-torsion, where $\varepsilon_{i}=\varepsilon_{i}(\varepsilon)$ and $q_{i}$ is projection to $T_{i}^{n}$. By our inductive hypothesis we have $[K, L] \in G_{i} \oplus \tilde{K}_{0}\left(T_{i}^{n} \times L\right)$, where $G_{i}$ is the group defined in the proof of Lemma 7.3. This implies that

because

$$
[K, L] \in \mathrm{Wh}(L) \oplus \sum_{i=1}^{n+1}\left(\begin{array}{c}
n+1 \\
i
\end{array}\right) \tilde{K}_{1-i}(L)
$$

$$
\bigcap_{i=1}^{n+1}\left[G_{i} \oplus \tilde{K}_{0}\left(T_{i}^{n} \times L\right)\right]=\mathrm{Wh}(L) \oplus \sum_{i=1}^{n+1}\left(\begin{array}{c}
n+1 \\
i
\end{array}\right) \tilde{K}_{1-i}(L) .
$$


8. Proof of Theorem 2. For $F$ a compact $Q$-manifold we want to establish a bijection $\sigma_{*}: \delta_{b}\left(R^{n} \times F\right) \approx \tilde{K}_{1-n}(F)$, for all $n \geqslant 1$. So our first task is to start with a bounded homotopy equivalence $f: M \rightarrow R^{n} \times F, M$ being a $Q$-manifold, and show how this gives rise to an element $\sigma(f) \in \tilde{K}_{1-n}(F)$. This will then induce our desired bijection $\sigma_{*}$. In what follows $p$ will denote projection to $R^{n}$ and $q$ will denote projection to $T^{n}$.

I. Definition of $\sigma(f)$. As in Lemma 5.1 we may regard $T^{n-1} \times R$ as an open subset of $\dot{B}_{3}^{n}$ so that $e^{n-1} \times$ id: $B_{2}^{n} \rightarrow B_{2}^{n}$ is the identity. Choose $K_{0}$ large and let $f_{0}^{\prime}$ : $M \rightarrow R^{n} \times F$ be defined by dividing the $R^{n}$-component of $f$ by $K_{0}$. That is, $f_{0}^{\prime}=\left(u_{0} \times \mathrm{id}\right) f$, where $u_{0}: R^{n} \rightarrow R^{n}$ is defined by $u_{0}(x)=x / K_{0}$. Clearly $f_{0}^{\prime}$ is a $p^{-1}(\delta)$-equivalence, for $\delta$ small. By Theorem 1 there is a $p^{-1}(\gamma)$-homotopy $f_{0}^{\prime} \simeq f_{0}^{\prime \prime}$ : $M \rightarrow R^{n} \times F$ for which $p f_{0}^{\prime \prime}$ is an approximate fibration. Define $f_{0}=\left(u_{0} \times \mathrm{id}\right)^{-1} f_{0}^{\prime \prime}:$ $M \rightarrow R^{n} \times F$. This is still an approximate fibration, but now we have a bounded homotopy

$$
f_{0}=\left(u_{0} \times \mathrm{id}\right)^{-1} f_{0}^{\prime \prime} \simeq\left(u_{0} \times \mathrm{id}\right)^{-1} f_{0}^{\prime}=f .
$$

Thus we have a bounded homotopy $f \simeq f_{0}$, where $f_{0}$ is a $p^{-1}(\delta)$-equivalence. If $\delta$ is small enough, then $f_{0}: f_{0}^{-1}\left(T^{n-1} \times R \times F\right) \rightarrow T^{n-1} \times R \times F$ is a $p^{-1}(\delta)$-equivalence over $T^{n-1} \times[-3,3] \times F$. By Theorem 4.2 there is a compact $Q$-manifold $\tilde{M}_{0}$ which contains $f_{0}^{-1}\left(T^{n-1} \times(-2,2) \times F\right)$ as an open set and a $q^{-1}(\varepsilon)$-equivalence $\tilde{f}_{0}: \tilde{M}_{0} \rightarrow T^{n} \times F$ so that the following rectangle commutes:

$$
\begin{array}{ccc}
\tilde{M}_{0} & \stackrel{\tilde{f}_{0}}{\rightarrow} & T^{n} \times F \\
\uparrow & & \uparrow \mathrm{id} \times e \times \mathrm{id} \\
f_{0}^{-1}\left(T^{n-1} \times(-2,2) \times F\right) & \stackrel{f_{0}}{\rightarrow} & T^{n-1} \times(-2,2) \times F
\end{array}
$$

Moreover, $\varepsilon$ can be made small by choosing $\delta$ small. By Lemma 7.4 we have the torsion $\tau\left(\tilde{f}_{0}\right)$ lying in the subgroup $\mathrm{Wh}(F) \oplus \sum_{i=1}^{n}\left(\begin{array}{c}n \\ i\end{array}\right) \tilde{K}_{1-i}(F)$ of $\mathrm{Wh}\left(T^{n} \times F\right)$. We define our desired $\sigma(f) \in \tilde{K}_{1-n}(F)$ to be the component of $\tau\left(\tilde{f}_{0}\right)$ in $\tilde{K}_{1-n}(F)$.

II. $\sigma(f)$ is well defined. First we show that $\sigma(f)$ is independent of the choice of $\tilde{f}_{0}$ : $\tilde{M}_{0} \rightarrow T^{n} \times F$. So suppose that we make another choice of a commutative rectangle:

$$
\begin{array}{ccc}
\bar{M}_{0} & \stackrel{\bar{f}_{0}}{\rightarrow} & T^{n} \times F \\
\uparrow & & \uparrow \mathrm{id} \times e \times \mathrm{id} \\
f_{0}^{-1}\left(T^{n-1} \times(-2,2) \times F\right) & \stackrel{f_{0}}{\rightarrow} & T^{n-1} \times(-2,2) \times F
\end{array}
$$

Again $\bar{M}_{0}$ is a compact $Q$-manifold and $\bar{f}_{0}$ is a $q^{-1}(\varepsilon)$-equivalence. We must show that $\tau\left(\tilde{f}_{0}\right)$ and $\tau\left(\bar{f}_{0}\right)$ have the same component in $\tilde{K}_{1-n}(F)$. Let

$$
N \subset f_{0}^{-1}\left(T^{n-1} \times(-2,2) \times F\right)
$$

be a compact $Q$-manifold which contains $f_{0}^{-1}\left(T^{n-1} \times[-1,1] \times F\right)$ and whose topological boundary is a bicollared $Q$-manifold. The given commutative rectangles, along with the Estimated Homotopy Extension Theorem, enable us to find a homotopy equivalence $g: \tilde{M} \rightarrow \bar{M}$ so that $g=$ id on $N, g \mid: \tilde{M}_{0}-\stackrel{\circ}{N} \rightarrow \bar{M}_{0}-\stackrel{\circ}{N}$ is a 
homotopy equivalence, and $\bar{f}_{0} g \simeq \tilde{f}_{0}$. Then $\tau(g)$ is just the inclusion-induced image of $\tau\left(g \mid \tilde{M}_{0}-\stackrel{N}{N}\right)$ in $\mathrm{Wh}\left(\bar{M}_{0}\right)$. If $\left(\bar{f}_{0}\right)_{*}: \mathrm{Wh}\left(\bar{M}_{0}\right) \rightarrow \mathrm{Wh}\left(T^{n} \times F\right)$ is the isomorphism induced by $\bar{f}_{0}$, then $\left(\bar{f}_{0}\right)_{*}(\tau(g))$ lies in the subgroup $\mathrm{Wh}\left(T^{n-1} \times F\right)$ of $\mathrm{Wh}\left(T^{n} \times F\right)$ which corresponds to the direct sum decomposition

$$
\mathrm{Wh}\left(T^{n} \times F\right)=\mathrm{Wh}\left(T^{n-1} \times F\right) \oplus \tilde{K}_{0}\left(T^{n-1} \times F\right) \oplus \operatorname{Nil}\left(T^{n-1} \times F\right) .
$$

The homotopy $\bar{f}_{0} g \simeq \tilde{f}_{0}$ gives us $\tau\left(\tilde{f}_{0}\right)-\tau\left(\bar{f}_{0}\right)=\left(\bar{f}_{0}\right)_{*}(\tau(g))$. Since $\tilde{K}_{1-n}(F)$ does not meet $\mathrm{Wh}\left(T^{n-1} \times F\right)$, it follows that the $\tilde{K}_{1-n}(F)$-components of $\tau\left(\tilde{f}_{0}\right)$ and $\tau\left(\bar{f}_{0}\right)$ are equal. Thus $\sigma(f)$ is independent of the choice of $\tilde{f}_{0}: \tilde{M}_{0} \rightarrow T^{n} \times F$.

We now show that $\sigma(f)$ is independent of the choice of $f_{0}: M \rightarrow R^{n} \times F$. So suppose that we have bounded homotopies $f \simeq f_{0}$ and $f \simeq f_{1}$, where $f_{0}$ and $f_{1}$ are $p^{-1}(\delta)$-equivalences. Choose $K$ large and let $u: R^{n} \rightarrow R^{n}$ be defined by $u(x)=$ $x / K$. Then $f_{0}^{\prime}=(u \times$ id $) f_{0}$ and $f_{1}^{\prime}=(u \times$ id $) f_{1}$ are $p^{-1}(\delta)$-equivalences for which $p f_{0}^{\prime}$ is close to $p f_{1}^{\prime}$. If we allow $K$ to take on values in the interval [1,K], then we get a proper homotopy $f_{0} \simeq f_{0}^{\prime}$ so that each level is a $p^{-1}(\delta)$-equivalence. Similarly we have a proper homotopy $f_{1}^{\prime} \simeq f_{1}$ so that each level is a $p^{-1}(\delta)$-equivalence. If we interpose a small homotopy from $f_{0}^{\prime}$ to $f_{1}^{\prime}$, then we get a proper homotopy $f_{t}: f_{0} \simeq f_{1}$ for which each level is a $p^{-1}(\delta)$-equivalence. Each $f_{t}$ is a $p^{-1}(\delta)$-equivalence which determines a $q^{-1}(\varepsilon)$-equivalence $\tilde{f}_{t}: \tilde{M}_{t} \rightarrow T^{n} \times F$ making the following rectangle commute:

$$
\begin{array}{ccc}
\tilde{M}_{t} & \tilde{f}_{t} & T^{n} \times F \\
\hat{\jmath} & & \uparrow \mathrm{id} \times e \times \mathrm{id} \\
f_{t}^{-1}\left(T^{n-1} \times(-2,2) \times F\right) & \stackrel{f_{t}}{\rightarrow} & T^{n-1} \times(-2,2) \times F
\end{array}
$$

Each torsion $\tau\left(f_{t}\right)$ has a component in $\tilde{K}_{1-n}(F)$, and all we need to do is show that all of these components are the same. Clearly it suffices to show that $\tau\left(\tilde{f}_{0}\right)$ and $\tau\left(\tilde{f}_{1}\right)$ have the same component in $\tilde{K}_{1-n}(F)$ provided that $p f_{0}$ is close to $p f_{1}$. We will actually show that $\tau\left(\tilde{f}_{0}\right)$ and $\tau\left(\tilde{f}_{1}\right)$ have the same component in the direct summand $\tilde{K}_{0}\left(T^{n-1} \times F\right)$ of $\mathrm{Wh}\left(T^{n} \times F\right)$ (which contains $\left.\tilde{K}_{1-n}(F)\right)$. The following pull-back diagrams give us proper homotopy equivalences $\bar{f}_{0}: \bar{M}_{0} \rightarrow T^{n-1} \times R \times F$ and $\bar{f}_{1}$ : $\bar{M}_{1} \rightarrow T^{n-1} \times R \times F:$

$$
\begin{array}{cccccc}
\bar{M}_{0} & \stackrel{\bar{f}_{0}}{\rightarrow} & T^{n-1} \times R \times F & \bar{M}_{1} & \stackrel{\tilde{f}_{1}}{\rightarrow} & T^{n-1} \times R \times F \\
\downarrow & & \downarrow \text { id } \times e \times \mathrm{id} \downarrow & & \downarrow \text { id } \times e \times \mathrm{id} \\
\tilde{M}_{0} & \stackrel{\tilde{f}_{0}}{\rightarrow} & T^{n} \times F & \tilde{M}_{1} & \stackrel{\tilde{f}_{1}}{\rightarrow} & T^{n} \times F
\end{array}
$$

By Proposition 4.7 of [16], the component of $\tau\left(\tilde{f}_{i}\right)$ in $\tilde{K}_{0}\left(T^{n-1} \times F\right)$ is just the splitting obstruction for the $Q$-manifold $\bar{M}_{i}$. Since $p f_{0}$ is close to $p f_{1}$,

$$
\left(\bar{f}_{0}\right)^{-1}\left(T^{n-1} \times(-1,1) \times F\right)
$$

embeds as an open subset of $\bar{M}_{1}$. This implies that the splitting obstructions for $\bar{M}_{0}$ 
and $\bar{M}_{1}$ coincide, ${ }^{5}$ and therefore $\sigma(f) \in \tilde{K}_{1-n}(F)$ is independent of the choice of $f_{0}$ : $M \rightarrow R^{n} \times F$.

III. Definition of $\sigma_{*}$. Define $\sigma_{*}: \delta_{b}\left(R^{n} \times F\right) \rightarrow \tilde{K}_{1-n}(F)$ by $\sigma_{*}([f])=\sigma(f)$. To show that this is well defined all we have left to do is prove that if $[f]=\left[f^{\prime}\right]$, then $\sigma(f)=\sigma\left(f^{\prime}\right)$. So assume that $f: M \rightarrow R^{n} \times F$ and $f^{\prime}: M^{\prime} \rightarrow R^{n} \times F$ are bounded homotopy equivalences, and let $h: M \rightarrow M^{\prime}$ be a homeomorphism for which $f^{\prime} h$ is boundedly homotopic to $f$. It follows that $\sigma(f)=\sigma\left(f^{\prime}\right)$ by a repetition of the argument of the preceding paragraph (because topological homeomorphisms preserve splitting obstructions). Thus $\sigma: \delta_{b}\left(R^{n} \times F\right) \rightarrow \tilde{K}_{1-n}(F)$ is well defined.

IV. $\sigma_{*}$ is one-to-one. It suffices to show that if $f: M \rightarrow R^{n} \times F$ is a bounded homotopy equivalence for which $\sigma(f)=0$, then $f$ is boundedly homotopic to a homeomorphism. Recall that $\sigma(f)$ is the component of $\tau\left(\tilde{f}_{0}\right)$ in $\tilde{K}_{1-n}(F)$, where $\tilde{f}_{0}$ : $\tilde{M}_{0} \rightarrow T^{n} \times F$ is a $q^{-1}(\varepsilon)$-equivalence for which the following diagram commutes:

$$
\begin{array}{ccc}
\tilde{M}_{0} & \stackrel{\tilde{f}_{0}}{\rightarrow} & T^{n} \times F \\
\text { open } \uparrow & & \\
f_{0}^{-1}\left(T^{n-1} \times(-2,2) \times F\right) & \stackrel{f_{0}}{\rightarrow} & T^{n-1} \times(-2,2) \times F
\end{array}
$$

Assertion. There is a compact $Q$-manifold $\bar{M}$ and a $q^{-1}(\varepsilon)$-equivalence $\bar{f}: \bar{M} \rightarrow T^{n}$ $\times F$ for which $\tau(\bar{f})=\sigma(f)$ and for which $\bar{f}=\tilde{f}_{0}$ over $e^{n}\left(B_{2}^{n}\right) \times F$.

Proof. By a mapping cylinder construction like that used at the beginning of $\$ 7$ we may assume that $\tilde{f}_{0}$ is a $q^{-1}(\varepsilon)$-strong deformation retraction and $T^{n} \times F$ is a $Z$-set in $\tilde{M}_{0}$. By the Relative Triangulation Theorem [6, p. 83] there is a compact polyhedral pair $\left(K, T^{n} \times L\right)$ and a homeomorphism $\tilde{M}_{0} \cong K \times Q$ for which $\{x\} \times F$ goes to $\{x\} \times L \times Q$, for each $x \in T^{n}$. So $\tilde{f}_{0}: K \times Q \rightarrow T^{n} \times L \times Q$ is a $q^{-1}(\varepsilon)$-strong deformation retraction. For $k$ large the composition

$$
r: K \times I^{k} \hookrightarrow K \times Q \stackrel{\tilde{f}_{0}}{\rightarrow} T^{n} \times L \times Q \stackrel{\text { proj }}{\rightarrow} T^{n} \times L
$$

is also a $q^{-1}(\varepsilon)$-strong deformation retraction. For any factorization $T^{n}=S_{i}^{1} \times$ $T_{i}^{n-1}$, torsions in the subgroup

$$
\mathrm{Wh}\left(T_{i}^{n-1} \times L\right) \cap\left[\mathrm{Wh}(L) \oplus \sum_{j=1}^{n-1}\left(\begin{array}{l}
n \\
j
\end{array}\right) \tilde{K}_{1-j}(L)\right]
$$

of Wh( $\left.T^{n} \times L\right)$ are realized by $q^{-1}(\varepsilon)$-strong deformation retractions to $T^{n} \times L$. By using the canonical method of injecting $\mathrm{Wh}\left(T_{i}^{n-1} \times L\right)$ into $\mathrm{Wh}\left(T^{n} \times L\right)$, which was described in $\$ 7$, we conclude that each element of the above subgroup can be represented by a torsion $\left[K^{\prime}, T^{n} \times L\right]$ for which there is a $q^{-1}(\varepsilon)$-strong deformation retraction $r^{\prime}: K^{\prime} \rightarrow T^{n} \times L$ such that $r^{\prime}=$ id over $e^{n}\left(B_{3}^{n}\right) \times L$. If $\tau$ is the component of $\tau(r)$ in $\mathrm{Wh}(L) \oplus \sum_{i=1}^{n-1}\left(\begin{array}{c}n \\ i\end{array}\right) \tilde{K}_{1-i}(L)$, then

$$
\tau \in \mathrm{Wh}\left(T_{i}^{n-1} \times L\right) \cap\left[\mathrm{Wh}(L) \oplus \sum_{j=1}^{n-1}\left(\begin{array}{l}
n \\
j
\end{array}\right) \tilde{K}_{1-j}(L)\right],
$$

\footnotetext{
${ }^{5}$ This is an easy exercise provided that the reader is armed with (1) the Triangulation Theorem for $Q$-manifolds, (2) the definition of the splitting obstruction for $\bar{f}_{i}: \bar{M}_{i} \rightarrow T^{n-1} \times R \times F$ (see [6, p. 69]), and (3) the engulfing result Lemma 3.2.
} 
for some $i$. Thus there exists a torsion $\left[K_{1}, T^{n} \times L\right]$ for which there is a $q^{-1}(\varepsilon)$ strong deformation retraction $r_{1}: K_{1} \rightarrow T^{n} \times L$ such that $\tau\left(r_{1}\right)=\tau(r)-\tau$ and $r_{1}=r$ over $e^{n}\left(B_{3}^{n}\right) \times L$.

Let $\bar{M}=K_{1} \times Q$ and consider the product

$$
f_{1}=r_{1} \times \mathrm{id}_{Q}: \bar{M} \rightarrow T^{n} \times F .
$$

(For convenience we identify the $Q$-factor in $\bar{M}$ with $Q_{k+1}$.) Then $\tau\left(f_{1}\right)=\sigma(f)$ and it is easy to get a $q^{-1}\left(\varepsilon_{1}\right)$-homotopy $f_{1} \simeq \bar{f}$ so that $\bar{f}=\tilde{f}_{0}$ over $e^{n}\left(B_{2}^{n}\right) \times F$, where $\varepsilon_{1}=\varepsilon_{1}(k)$. If $k$ is large enough, then $\bar{f}$ is still a $q^{-1}(\varepsilon)$-equivalence.

Since $\sigma(f)=0, \bar{f}$ is homotopic to a homeomorphism $h: \bar{M} \rightarrow T^{n} \times F$. Now form the following pull-back diagram:

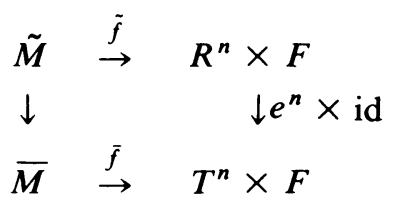

Recalling that $e^{n-1} \times$ id: $B_{2}^{n} \rightarrow B_{2}^{n}$ is the identity we can choose notation so that $\tilde{f}=f_{0}$ over $\dot{B}_{2}^{n} \times F$. Also there is a bounded homotopy of $\tilde{f}$ to a homeomorphism $\tilde{h}$. Moreover it follows from Proposition 2.1 that $\tilde{f}$ is a $p^{-1}(\tilde{\varepsilon})$-equivalence, where $\tilde{\varepsilon}=\tilde{\varepsilon}(\varepsilon)$ is small if $\varepsilon$ is small. Choose $K>0$ large and let $f_{1}: \tilde{M} \rightarrow R^{n} \times F$ be defined so that (i) it agrees with $\tilde{f}$ over $B_{K-1}^{n} \times F$, (ii) it agrees with $\tilde{h}$ over $\left(R^{n}-\dot{B}_{K}^{n}\right) \times F$, and (iii) over $\left(B_{K}^{n}-\dot{B}_{K-1}^{n}\right) \times F$ it is defined by using the bounded homotopy $\tilde{h} \simeq \tilde{f}$. (For this it is convenient to assume that

$$
\tilde{h}^{-1}\left(\left(R^{n}-\dot{B}_{K}^{n}\right) \times F\right) \text { and } \tilde{f}^{-1}\left(B_{K-1}^{n} \times F\right)
$$

are disjoint.) We now refer the reader to the last half of the proof of Lemma 5.1. Specifically, we refer to the part which follows the proof of the assertion. Repeating this construction it is easy to get an open embedding $g: f_{0}^{-1}\left(\dot{B}_{2}^{n} \times F\right) \rightarrow R^{n} \times F$ which is $p^{-1}\left(\varepsilon_{1}\right)$-homotopic to $f_{0} \mid f_{0}^{-1}\left(\dot{B}_{2}^{n} \times F\right)$, for some small $\varepsilon_{1}>0$. Then we obtain a $p^{-1}(\bar{\delta})$-equivalence $\bar{f}_{0}: M \rightarrow R^{n} \times F$ which is a homeomorphism over $\dot{B}_{1.5}^{n} \times F$ and which is boundedly homotopic to $f_{0}$.

To finish the proof we will show that there is a bounded homotopy of $\bar{f}_{0}$ to a homeomorphism. Since $\bar{f}_{0}$ is a $p^{-1}(\bar{\delta})$-equivalence, $p \bar{f}_{0}: M \rightarrow R^{n}$ is easily seen to be a $2 \bar{\delta}$-fibration. Also $p \bar{f}_{0}$ is an $\alpha$-fibration over $\dot{B}_{1.5}^{n}$, for every open cover $\alpha$ of $\dot{B}_{1.5}^{n}$. It follows from Theorem 1 that if $\bar{\delta}$ is sufficiently small, then there is a small homotopy of $p \bar{f}_{0} \operatorname{rel}\left(p \bar{f}_{0}\right)^{-1}\left(B_{1.4}^{n}\right)$ to an approximate fibration $p^{\prime}: M \rightarrow R^{n}$. The small homotopy $p \bar{f}_{0} \simeq p^{\prime}$ lifts to a homotopy $\bar{f}_{0} \simeq f^{1} \operatorname{rel}\left(\bar{f}_{0}\right)^{-1}\left(B_{1.4}^{n} \times F\right)$. Thus $f^{1}$ is a homeomorphism over $B_{1.2}^{n} \times F$ and $p f^{1}=p^{\prime}$ is an approximate fibration. By Proposition $2.3, f^{1}$ is a $p^{-1}(\alpha)$-equivalence, for every open cover $\alpha$ of $R^{n}$.

Again using the ideas of the last half of the proof of Lemma 5.1 we can find homeomorphisms $\theta: R^{n} \rightarrow R^{n}, \tilde{\theta}: M \rightarrow M$ so that $f^{2}=(\theta \times \text { id })^{-1} f^{1} \tilde{\theta}: M \rightarrow R^{n} \times$ $F$ is a homeomorphism over $B_{2.2}^{n} \times F$ and so that $f^{2} \simeq f^{1} \operatorname{rel}\left(f^{1}\right)^{-1}\left(B_{1}^{n} \times F\right){ }^{6}$ Moreover, the $R^{n}$-component of this homotopy can be made as small as we please

\footnotetext{
${ }^{6}$ The map $\theta$ is a radially-defined contraction which takes $B_{2.2}^{n}$ to $B_{1.2}^{n}$, and $\theta=$ id on $B_{1.1}^{n}$.
} 
by making an appropriate choice of $\tilde{\theta}$. Note that $f^{2}$ is still a $p^{-1}(\alpha)$-equivalence, for every open cover $\alpha$ of $R^{n}$. Repeating this construction we get

$$
f^{2} \simeq f^{3} \operatorname{rel}\left(f^{2}\right)^{-1}\left(B_{2}^{n} \times F\right)
$$

so that $f^{3}$ is a homeomorphism over $B_{3.2}^{n} \times F, f^{3}$ is a $p^{-1}(\alpha)$-equivalence for every $\alpha$, and so that the $R^{n}$-component of this homotopy is small. It is then clear that we can continue this process to get our desired bounded homotopy of $f^{1}$ to the homeomorphism $\lim _{n \rightarrow \infty} f^{n}$.

V. $\sigma_{*}$ is onto. Choose any torsion $\tau \in \tilde{K}_{1-n}(F) \subset \mathrm{Wh}\left(T^{n} \times F\right)$ and let $f_{1}$ : $M_{1} \rightarrow T^{n} \times F$ be a homotopy equivalence of a compact $Q$-manifold to $T^{n} \times F$ whose torsion is $\tau$. By Lemma 7.3 we can choose $f_{1}$ to be a $q^{-1}\left(\varepsilon_{1}\right)$-equivalence, where $\varepsilon_{1}$ is small. The pull-back diagram,

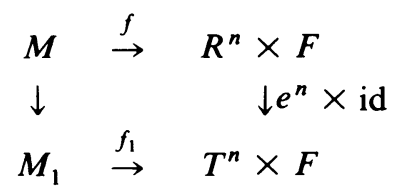

gives us a $p^{-1}(\delta)$-equivalence, where $\delta$ is small. We will prove that $\sigma(f)=\tau$.

Let $\tilde{f}: \tilde{M} \rightarrow T^{n} \times F$ be a $q^{-1}(\varepsilon)$-equivalence for which the following diagram commutes ( $\varepsilon$ is small):

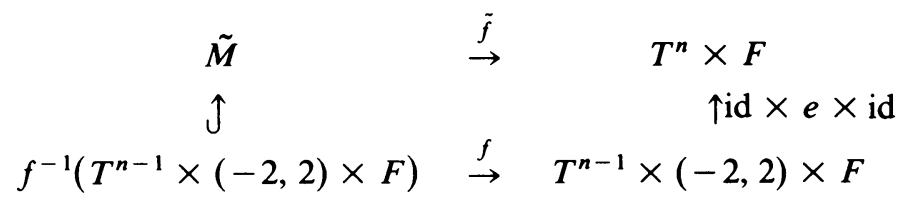

Then $\sigma(f)$ is the component of $\tau(\tilde{f})$ in $\tilde{K}_{1-n}(F)$. Since $e^{n-1} \times$ id: $B_{2}^{n} \rightarrow B_{2}^{n}$ is the identity we can choose notation so that $\tilde{f}=f_{1}$ over $e^{n}\left(\dot{B}_{2}^{n}\right) \times F$. Recalling the proof of the assertion in step IV above we can represent $\tilde{f}$ and $f_{1}$ by $q^{-1}(\varepsilon)$-strong deformation retractions $\tilde{r}: \tilde{K} \rightarrow T^{n} \times L, r_{1}: K_{1} \rightarrow T^{n} \times L$ so that $\tilde{r}=r_{1}$ over $e^{n}\left(B_{1}^{n}\right) \times L$. We need to show that $\tau(\tilde{r})$ and $\tau\left(r_{1}\right)$ have the same components in $\tilde{K}_{1-n}(L)$. Let $r_{2}: K_{2} \rightarrow T^{n} \times L$ be a strong deformation retraction whose torsion is $-\tau\left(r_{1}\right)$. We assume that $K_{2}$ is constructed as on p. 21 of [8], and for convenience we assume that $r_{2}$ is a $q^{-1}(\varepsilon)$-strong deformation retraction. Then $\tilde{r}$ and $r_{2}$ piece together to give us a $q^{-1}(\varepsilon)$-strong deformation retraction $\bar{r}: \bar{K}=\tilde{K} \cup K_{2} \rightarrow T^{n} \times$ $L$ whose torsion is $\tau(\tilde{r})-\tau\left(r_{1}\right)$. So all we have to do is prove that the component of $\tau(\vec{r})$ in $\tilde{K}_{1-n}(L)$ is 0 .

On p. 21 of [8] there is given a geometric proof that $\left[K_{1}, T^{n} \times L\right]+\left[K_{2}, T^{n} \times\right.$ $L]=0$. If we localize this argument over $e^{n}\left(B_{1}^{n}\right) \times L$ and use the fact that $\tilde{r}=r_{1}$ over $e^{n}\left(B_{1}^{n}\right) \times L$, then we get a strong deformation retraction $\hat{r}: \hat{K} \rightarrow T^{n} \times L$ which is the identity over $e^{n}\left(B_{1 / 2}^{n}\right) \times L$ and whose torsion is $\tau(\vec{r})$. For convenience we assume that $\hat{r}$ is a $q^{-1}(\varepsilon)$-strong deformation retraction. By the proof of the assertion in step IV we may assume that $\tau(\hat{r}) \in \tilde{K}_{1-n}(L)$. So we want to prove that $\tau(\hat{r})=0$. If $n=1$, the proof is easy. The condition $\hat{r}=\mathrm{id}$ over $e\left(\left[-\frac{1}{2}, \frac{1}{2}\right]\right) \times L$ means that we must have $\tau(\hat{r})$ lying in the subgroup $\mathrm{Wh}(L)$ of $\mathrm{Wh}\left(T^{1} \times L\right)$. By our assumption that $\tau(\hat{r}) \in \tilde{K}_{0}(L)$ we must have $\tau(\hat{r})=0$. 
Proceeding by induction, choose a factorization $T^{n}=S^{1} \times T^{n-1}$. The canonical retraction $\mathrm{Wh}\left(T^{n} \times L\right) \rightarrow \mathrm{Wh}\left(T^{n-1} \times L\right)$ sends the $q^{-1}(\varepsilon)$-equivalence $\hat{r}: \hat{K} \rightarrow T^{n}$ $\times L$ to the $q_{1}^{-1}(\varepsilon)$-equivalence $u: J \rightarrow T^{n-1} \times L$, where $J=\hat{K} \cup\left(D^{2} \times T^{n-1} \times\right.$ $L), T^{n-1} \times L \equiv\{1\} \times T^{n-1} \times L$, and $u$ is the composition

$$
J^{\hat{r} \cup \text { id }} \rightarrow D^{2} \times T^{n-1} \times L \stackrel{\text { retraction }}{\rightarrow} T^{n-1} \times L .
$$

By our assumption that $\tau(\hat{r}) \in \tilde{K}_{1-n}(L)$ we have $\tau(u)=0$. Thus step IV implies that there is an open embedding $g: u^{-1}\left(e^{n-1}\left(\dot{B}_{2}^{n-1}\right) \times L\right) \times Q \rightarrow T^{n-1} \times L \times Q$ which is homotopic to $u \times \mathrm{id} \mid u^{-1}\left(e^{n-1}\left(\dot{B}_{2}^{n-1}\right) \times L\right) \times Q$ via a homotopy whose $T^{n-1}$-component is small.

Let $A=S^{1}-e((-1 / 3,1 / 3))$ and consider

$$
\begin{aligned}
u^{-1}\left(e^{n-1}\left(\dot{B}_{1 / 3}^{n-1}\right) \times L\right) & \\
= & (\hat{r})^{-1}\left(S^{1} \times e^{n-1}\left(\check{B}_{1 / 3}^{n-1}\right) \times L\right) \cup\left(D^{2} \times e^{n-1}\left(\dot{B}_{1 / 3}^{n-1}\right) \times L\right) .
\end{aligned}
$$

By collapsing the $D^{2}$-factor to $A$ we obtain a collapse of $u^{-1}\left(e^{n-1}\left(B_{1 / 3}^{n-1}\right) \times L\right)$ to $(\hat{r})^{-1}\left(A \times e^{n-1}\left(B_{1 / 3}^{n-1}\right) \times L\right)$. By the CE Approximation Theorem of [3] this collapse stabilizes to a near homeomorphism upon multiplication by $Q$. Since $A \times Q$ is homeomorphic to $Q$ we obtain a homeomorphism $T^{n-1} \times L \times Q \cong A \times T^{n-1}$ $\times L \times Q$. Combining these homeomorphisms with the open embedding $g$ we obtain an open embedding $g_{1}:(\hat{r})^{-1}\left(A \times e^{n-1}\left(B_{1 / 4}^{n-1}\right) \times L\right) \times Q \rightarrow A \times$ $e^{n-1}\left(\dot{B}_{1 / 3}^{n-1}\right) \times L \times Q$ which is homotopic to $\left(\hat{r} \times \mathrm{id}_{Q}\right) \mid$ via a homotopy whose $T^{n-1}$-component is small. By Proposition 2.4 we may assume that $g_{1}=$ id on $\partial A \times e^{n-1}\left(B_{1 / 4}^{n-1}\right) \times L \times Q$ and that the homotopy $g_{1} \simeq(\hat{r} \times$ id $) \mid$ is rel $\partial A \times$ $e^{n-1}\left(\dot{B}_{1 / 4}^{n-1}\right) \times L \times Q$. Thus $g_{1}$ extends via the identity to an open embedding

$$
\tilde{g}_{1}:(\hat{r})^{-1}\left(S^{1} \times e^{n-1}\left(\dot{B}_{1 / 4}^{n-1}\right) \times L\right) \times Q \rightarrow S^{1} \times e^{n-1}\left(\dot{B}_{1 / 3}^{n-1}\right) \times L \times Q
$$

which is homotopic to $\left(\hat{r} \times\right.$ id) $\mid$ via a homotopy whose $T^{n-1}$-component is small. Therefore we can deform $\hat{r} \times \mathrm{id}_{Q}$ to a map $w: \hat{K} \times Q \rightarrow T^{n} \times L \times Q$ which is a homeomorphism over $S^{1} \times e^{n-1}\left(B_{1 / 5}^{n-1}\right) \times L \times Q$. Moreover, the $T^{n-1}$-component of this homotopy is small, so $w$ is a $q_{1}^{-1}\left(\varepsilon_{1}\right)$-equivalence (for $\varepsilon_{1}$ small).

Refactor $T^{n} \times L \times Q$ as $T^{n} \times L \times Q=T^{n-1} \times\left(S^{1} \times L \times Q\right)$, where $S^{1} \times$ $L \times Q$ is now regarded as the fiber. Since $\tau(w) \in \tilde{K}_{1-n}(L \times Q)$ we conclude that $\tau(w) \in \tilde{K}_{1-(n-1)}\left(S^{1} \times L \times Q\right) \subset \mathrm{Wh}\left(T^{n-1} \times\left(S^{1} \times L \times Q\right)\right)$. We are inductively assuming that any $q^{-1}(\varepsilon)$-strong deformation retraction to $T^{n-1} \times F^{\prime}$ must have 0 -torsion provided that that torsion lies in $\tilde{K}_{1-(n-1)}\left(F^{\prime}\right)$. So setting $F^{\prime}=S^{1} \times L \times$ $Q$ we conclude that $\tau(w)=0$ as desired.

9. Proof of Theorem 3. Proceeding in the spirit of the proof of Theorem 1 we treat the following cases: $B$ is a polyhedron, $B$ is a $Q$-manifold, and $B$ is an ANR.

I. $B$ is a polyhedron. For $B$ compact we proceed by induction on $\operatorname{dim} B$. Once this has been done, the noncompact case follows quickly from the proof of the compact case (just as in the proof of Theorem 1). For the case $\operatorname{dim} B=0$ we might as well assume that $B=$ \{point $\}$. Then we are dealing with a homotopy equivalence between compact $Q$-manifolds, $f: M \rightarrow E$. Since $\mathscr{F}$ is homotopy equivalent to 
$E$ we have $\mathrm{Wh}(E)=0$. Thus $\tau(f)=0$ and $f$ is homotopic to a homeomorphism as desired.

For the inductive step it suffices to consider the case $B=B_{1} \cup \Delta^{n}$, where $\operatorname{dim} B_{1}=n-1$ and $\Delta^{n}$ is the single principal $n$-cell in $B$. Assuming the result to be true for $B_{1}$, we prove that it is also true for $B$. For notation we identify $\bigsqcup^{n}$ with $R^{n}$. Let $q: \mathcal{E} \rightarrow B$ be a Hurewicz fibration for which there is a homotopy equivalence $h: E \rightarrow \mathcal{E}$ such that $q h \simeq p$ [17]. Using the homotopy lifting property we may assume that $q h=p$. By Proposition 2.3 we conclude that $h$ is a $q^{-1}\left(\beta^{\prime}\right)$-equivalence, where $\beta^{\prime}$ is as fine as we want.

Now choose a compact $Q$-manifold $N$ for which $p^{-1}\left(B_{1}^{n}\right) \subset N \subset p^{-1}\left(B_{2}^{n}\right)$. If $\beta^{\prime}$ is sufficiently fine, then the map $h \mid: N \rightarrow q^{-1}\left(\Delta^{n}\right)$ must be a homotopy domination. Thus $\mathscr{F}$ (which is homotopy equivalent to $q^{-1}\left(\Delta^{n}\right)$ ) is finitely dominated by $N$. Since $\tilde{K}_{0}(\mathscr{F})=0$ we conclude that $\mathscr{F}$ is homotopy equivalent to some compact polyhedron. (We are using the finiteness obstruction of Wall [18].) Thus $\mathscr{F}$ is homotopy equivalent to a compact $Q$-manifold $F$. It follows that there is a fiber homotopy equivalence $q^{-1}\left(\Delta^{n}\right) \simeq \Delta^{n} \times F$. By this we may assume that $q^{-1}\left(\Delta^{n}\right)$ is identified with the trivial fibration $\Delta^{n} \times F$.

Since $\tilde{K}_{1-n}(F)=0$ we can use Theorem 2 to deform $h$ slightly (measured in $B$ ) to $\tilde{h}: E \rightarrow \mathcal{E}$ so that $\tilde{h}$ is a homeomorphism over $B_{3}^{n} \times F$. (Step IV of Theorem 2 explains why Theorem 2 applies here.) Thus we can make an identification of $p \mid$ : $p^{-1}\left(B_{2}^{n}\right) \rightarrow B_{2}^{n}$ with proj: $B_{2}^{n} \times F \rightarrow B_{2}^{n}$. Again using Theorem 2 we can deform $f$ slightly to $\tilde{f}: M \rightarrow E$ so that it is a homeomorphism over $B_{1.5}^{n} \times F$.

Let $B_{0}=B-\dot{B}_{1}^{n}, E_{0}=E-\left(\dot{B}_{1}^{n} \times F\right)$, and $M_{0}=M-\tilde{f}^{-1}\left(\dot{B}_{1}^{n} \times F\right)$. Restricting $\tilde{f}$ and $p$ we obtain maps $f_{0}: M_{0} \rightarrow E_{0}$ and $p_{0}: E_{0} \rightarrow B_{0}$. It follows from Proposition 2.2 that $p_{0}$ is a $\beta_{0}$-fibration and it follows from Proposition 2.1 that $f_{0}$ is a $p_{0}^{-1}\left(\beta_{0}\right)$-equivalence, where $\beta_{0}$ is a fine open cover of $B_{0}$. Also $f_{0}$ is a homeomorphism over $\partial B_{1}^{n} \times F$ and $f_{0}^{-1}\left(\partial B_{1}^{n} \times F\right)$ is a $Z$-set in $M_{0}$. Let $r: B_{0} \rightarrow B_{1}$ be the retraction induced by the radially-defined retraction of $\Delta^{n}-\dot{B}_{1}^{n}$ to $\partial \Delta^{n}$. We leave it as an easy exercise to show that $r p_{0}: E_{0} \rightarrow B_{1}$ is a $\beta_{1}$-fibration and $f_{0}: M_{0} \rightarrow E_{0}$ is a $\left(r p_{0}\right)^{-1}\left(\beta_{1}\right)$-equivalence, for $\beta_{1}$ a fine open cover of $B_{1}$. (This should be compared with the proof of Assertion 1 in step II of the proof of Theorem 1.) By the inductive hypothesis $f_{0}$ is homotopic to a homeomorphism $g_{0}: M_{0} \rightarrow E_{0}$. Moreover, the composition of this homotopy with $r p_{0}$ is small, and by Proposition 2.4 we may take this homotopy to be $\operatorname{rel}_{0}^{-1}\left(\partial B_{1}^{n} \times F\right)$. Then we obtain a homeomorphism $g=g_{0} \cup$ id: $M \rightarrow E$ which is homotopic to $f$. Also the composition of this homotopy with $p$ is small provided that we choose notation so that $B_{1}^{n}$ is very nearly all of $\Delta^{n}$. This completes the polyhedral case.

II. $B$ is a $Q$-manifold. The proof goes somewhat like the proof of a corresponding step in Theorem 1. Choose a factorization, $B=B_{1} \times Q$, where $B_{1}$ is a polyhedron and so that each $\{x\} \times Q$ is small with respect to the open cover $\alpha$. Let $p_{1}=$ proj: $B \rightarrow B_{1}$. Following the proof of Theorem 1, $p_{1} p: E \rightarrow B_{1}$ is a $\beta_{1}$-fibration, where $\beta_{1}$ is a fine open cover of $B_{1}$. It also easily follows that $f: M \rightarrow E$ is a $\left(p_{1} p\right)^{-1}\left(\beta_{1}\right)$ equivalence.

By the polyhedral case, $f$ is homotopic to a homeomorphism via a homotopy 
which is small when composed with $p_{1} p$. By the choice of $p_{1}$, this homotopy is an $\alpha$-homotopy when composed with $p$. Thus $f$ is $p^{-1}(\alpha)$-homotopic to a homeomorphism as desired.

III. $B$ is an $A N R$. Multiplication by $Q$ yields a $\beta_{1}$-fibration $p \times$ id: $E \times Q \rightarrow B$ $\times Q$ and a $(p \times \text { id })^{-1}\left(\beta_{1}\right)$-equivalence $f \times$ id: $M \times Q \rightarrow E \times Q$, where $\beta_{1}$ is a fine open cover of $B \times Q$. Then there is a $(p \times \text { id })^{-1}\left(\alpha_{1}\right)$-homotopy of $f \times$ id to a homeomorphism $h_{1}: M \times Q \rightarrow E \times Q$ by the $Q$-manifold case above. If $u$ : $M \times Q \rightarrow M$ and $v: E \times Q \rightarrow E$ are homeomorphisms which are close to projection, then

$$
h: M \stackrel{u^{-1}}{\rightarrow} M \times Q \stackrel{h_{1}}{\rightarrow} E \times Q \stackrel{v}{\rightarrow} E
$$

is a homeomorphism which is $\alpha$-homotopic to $f$.

10. Proof of Theorem 4. We first expand slightly on the definition of a block bundle which was given in $\S 1$. Let $B$ be a polyhedron for which $B=|K|$ (i.e., $B$ is triangulated by the simplicial complex $K$ ). Then following the definition given in $\S 1$ we say that $p: E \rightarrow B$ is a $K$-block bundle with fiber $F$ provided that for each $\sigma \in K$ there is a $K$-block preserving homeomorphism $p^{-1}(\sigma) \cong \sigma \times F$. (Recall that $E$ is a $Q$-manifold and $F$ is a compact $Q$-manifold.) When the meaning is clear we drop the prefix $K$.

LeMma 10.1. Let $p: E \rightarrow B$ be a $K$-block bundle with fiber $F$ such that $K$ is a finite complex which simplicially collapses to a point. Then there is a $K$-block preserving homeomorphism $E \cong B \times F$.

Proof. Let $\Delta^{n} \in K$ be an $n$-cell, let $\sigma^{n-1} \subset \Delta^{n}$ be a face, and let $\delta=\partial \Delta-\stackrel{\circ}{\text { (the }}$ closure of $\partial \Delta-\sigma)$. Assume that $B=B_{1} \cup \Delta$, where $B_{1}=\left|K_{1}\right|$, for $K_{1}$ a collapsible subcomplex of $K$ and $B_{1} \cap \Delta=\delta$. It will suffice to prove that any block preserving homeomorphism $h_{1}: p^{-1}\left(B_{1}\right) \cong B_{1} \times F$ extends to a block preserving homeomorphism $h: E \cong B \times F$. (We are inducting on the reverse of the collapse.)

Let $h_{2}: p^{-1}(\Delta) \rightarrow \Delta \times F$ be a block preserving homeomorphism. Then $h_{1} h_{2}^{-1}$ : $\delta \times F \rightarrow \delta \times F$ is block preserving. Let $D^{n}$ be the $n$-disc with boundary $S^{n-1}$ and write $S^{n-1}=D_{1}^{n-1} \cup D_{2}^{n-1}$, where $D_{1}^{n-1}$ and $D_{2}^{n-1}$ are the upper and lower hemispheres. Then we clearly have a homeomorphism of triples,

$$
(\Delta \times F, \delta \times F, \sigma \times F) \cong\left(D^{n} \times F, D_{1}^{n-1} \times F, D_{2}^{n-1} \times F\right) .
$$

This implies that $h_{1} h_{2}^{-1} \mid \delta \times F$ extends to a block preserving homeomorphism $\theta$ : $\Delta \times F \rightarrow \Delta \times F$. Then $\tilde{h}_{2}=\theta h_{2}: p^{-1}(\Delta) \rightarrow \Delta \times F$ is block preserving and it agrees with $h_{1}$ on $p^{-1}(\delta)$. Thus $h_{1}$ and $\tilde{h}_{2}$ piece together to give a block preserving homeomorphism $E \cong B \times F$.

REMARK. Lemma 10.1 implies the following interesting fact concerning the definition of a block bundle $p: E \rightarrow B$ : If we drop the requirement that $E$ be $a$ $Q$-manifold, we can prove that $E$ must be a $Q$-manifold. (Because each point in $B$ has a collapsible neighborhood.) 
LemMA 10.2. Let $p: E \rightarrow B$ be a $K$-block bundle with fiber $F$ such that $K$ is a finite complex. If $K^{\prime}$ is the first barycentric subdivision of $K$, then there is a $K^{\prime}$-block bundle $p^{\prime}: E \rightarrow B$ and $a K$-block preserving homotopy $p^{\prime} \simeq p$.

Proof. It suffices to construct $p^{\prime}: E \rightarrow B$ by inducting on the skeleta. The inductive step reduces to the following special case: Let $B=\Delta^{n}$, the standard $n$-simplex with standard triangulation $K$ consisting of $n+1$ vertices, and let $K^{\prime}$ be the first barycentric subdivision of $K$. Assume that $p: E \rightarrow B$ is a $K$-block bundle for which $p \mid: p^{-1}(\partial \Delta) \rightarrow \partial \Delta$ is already a $K^{\prime}$-block bundle. We want to prove that $p$ is homotopic to $p^{\prime}: E \rightarrow B$ rel $p^{-1}(\partial \Delta)$, where $p^{\prime}$ is a $K^{\prime}$-block bundle.

Let $\sigma \subset \partial \Delta$ be an $(n-1)$-simplex of $K^{\prime}$ and let $\delta=\partial \Delta-\stackrel{\circ}{\sigma}$. Also let $\tilde{\sigma} \subset \partial \Delta$ be an $(n-1)$-simplex of $K$ containing $\sigma$ and let $\tilde{\delta}=\partial \Delta-(\tilde{\sigma})^{\circ}$. If $\sigma_{1} \subset \partial \Delta$ is an $(n-1)$-simplex of $K^{\prime}$ missing $\tilde{\sigma}$ and $\delta_{1}=\partial \Delta-\circ_{1}$, then Lemma 10.1 gives us a $K^{\prime}$-block preserving homeomorphism $p^{-1}\left(\delta_{1}\right) \cong \delta_{1} \times F$. Clearly there exists a homeomorphism of $\delta_{1} \times F$ onto itself which is the identity on $\partial \delta_{1} \times F$, which takes $\sigma \times F$ to $\tilde{\sigma} \times F$, and which is homotopic to id rel $\partial \delta_{1} \times F$. This induces a homeomorphism $\theta: p^{-1}\left(\delta_{1}\right) \rightarrow p^{-1}\left(\delta_{1}\right)$ which extends via the identity to a homeomorphism $\tilde{\theta}: p^{-1}(\partial \Delta) \rightarrow p^{-1}(\partial \Delta)$ for which (1) $\tilde{\theta} \simeq$ id and (2) $\tilde{\theta}$ takes $p^{-1}(\sigma)$ to $p^{-1}(\tilde{\sigma})$. By $Z$-set unknotting $\tilde{\theta}$ extends to a homeomorphism $\hat{\theta}: E \rightarrow E$ which takes $p^{-1}(\sigma)$ to $p^{-1}(\tilde{\sigma})$. We observed in the proof of Lemma 10.1 that there is a homeomorphism of triples, $(\Delta \times F, \tilde{\delta} \times F, \tilde{\sigma} \times F) \cong\left(D^{n} \times F, D_{1}^{n-1} \times F, D_{2}^{n-1}\right.$ $\times F)$. The given $K$-block preserving homeomorphism $E \simeq \Delta \times F$ then gives us a homeomorphism of triples, $\left(E, p^{-1}(\tilde{\delta}), p^{-1}(\tilde{\sigma})\right) \cong\left(D^{n} \times F, D_{1}^{n-1} \times F, D_{2}^{n-1} \times\right.$ $F)$. Using the homeomorphism $\hat{\theta}$ we get a homeomorphism of triples, $\left(E, p^{-1}(\delta), p^{-1}(\sigma)\right) \cong\left(D^{n} \times F, D_{1}^{n-1} \times F, D_{2}^{n-1} \times F\right)$. Also there is a homeomorphism of triples, $(\Delta \times F, \delta \times F, \sigma \times F) \cong\left(D^{n} \times F, D_{1}^{n-1} \times F, D_{2}^{n-1} \times F\right)$. If $g: p^{-1}(\delta) \rightarrow \delta \times F$ is a $K^{\prime}$-block preserving homeomorphism, which exists by Lemma 10.1, then the above homeomorphisms of triples give us a homeomorphism $h: E \rightarrow \Delta \times F$ which extends $g$ and which is $K^{\prime}$-block preserving over $\partial \Delta$.

Clearly there is a $K^{\prime}$-block preserving homotopy $p\left|p^{-1}(\partial \Delta) \simeq \operatorname{proj}_{\Delta} \circ h\right| p^{-1}(\partial \Delta)$. This homotopy is constructed inductively over the skeleta in $\partial \Delta$. Define $p^{\prime}: E \rightarrow \Delta$ by $p^{\prime}=p$ on $p^{-1}(\partial \Delta), p^{\prime}=\operatorname{proj}_{\Delta} \circ h$ on $h^{-1}\left(B_{1}^{n} \times F\right)$ (where $\left.\doteq=R^{n}\right)$, and $p^{\prime}$ on $h^{-1}\left(\left(\Delta-\dot{B}_{1}^{n}\right) \times F\right)$ is defined by the homotopy $p\left|p^{-1}(\partial \Delta) \simeq \operatorname{proj}_{\Delta} \circ h\right| p^{-1}(\partial \Delta)$. (We are choosing $B_{1}^{n}$ in $\Delta$ so that if $\partial \Delta \times[0,1] \subset \Delta$ is a $K^{\prime}$-block preserving collaring with $\partial \Delta \times\{0\} \equiv \partial \Delta$, then $B_{1}^{n}=\Delta-\left(\partial \Delta \times\left[0, \frac{1}{2}\right)\right)$.) Then it is easy to see that $p^{\prime}: E \rightarrow \Delta$ is a $K^{\prime}$-block bundle which is homotopic to $p$ rel $p^{-1}(\partial \Delta)$.

We now combine Lemmas 10.1 and 10.2 to establish a result which was mentioned in $\S 1$.

Proposition 10.3. If $p: E \rightarrow B$ is a block bundle, then there is a block preserving homotopy of $p$ to a new block bundle which is an approximate fibration.

Proof. First assume that $B$ is compact and let $B=|K|$. If $K^{(n)}$ is the $n$th barycentric subdivision of $K$, then iterating Lemma 10.2 there is a $K$-block preserving homotopy $p \simeq p^{\prime}$ such that $p^{\prime}$ is a $K^{(n)}$-block bundle. We will show that if $\varepsilon>0$ is given, then for a sufficiently large choice of $n$ the map $p^{\prime}$ must be an 
$\varepsilon$-fibration. Iteration of this result gives our desired homotopy of $p$ to an approximate fibration (compare with the proof of Theorem 1).

Recall from Proposition 2.2 that $\varepsilon$-fibrations are detected locally. For each point $b \in B$ there is a $K$-collapsible neighborhood $N \subset B$ of $b$. So all we have to do is prove that $p^{\prime}$ has the $\varepsilon$-lifting property over $N$. Since $N$ is also $K^{(n)}$-collapsible we can use Lemma 10.1 to get a $K^{(n)}$-block preserving homeomorphism $\left(p^{\prime}\right)^{-1}(N) \cong$ $N \times F$. This implies that $p^{\prime}$ has the $\varepsilon$-lifting property over $N$ for $n$ large.

Having done the compact case the noncompact case follows routinely (as in the proofs of Theorems 1 and 3).

REMARK. It follows from the above proof that if $p^{\prime}: E \rightarrow B$ is the new block bundle which is an approximate fibration, then $\left(p^{\prime}\right)^{-1}(\sigma)=p^{-1}(\sigma)$, for all simplices $\sigma$ in $B$.

Lemma 10.4. Let $q: \mathcal{E} \rightarrow B$ be a Hurewicz fibration over a polyhedron whose fiber has the homotopy type of a compact $Q$-manifold $F$ for which $\mathrm{Wh}(F)=0$. Then there exist a block bundle $p: E \rightarrow B$ with fiber $F$ and a homotopy equivalence $f: E \rightarrow \mathcal{E}$ which is block preserving.

Proof. In what follows below we will construct $p: E \rightarrow B$ and a block preserving map $f: E \rightarrow \mathcal{E}$ so that for each vertex $b$ of $B$, the restriction $f \mid p^{-1}(b): p^{-1}(b) \rightarrow$ $q^{-1}(b)$ is a homotopy equivalence. Then all we need is the

Assertion. $f$ is a homotopy equivalence, in fact, a block preserving homotopy equivalence.

Proof. By Proposition 10.3 we may assume that $p: E \rightarrow B$ is an approximate fibration. Since $f$ is block preserving we have $q f \simeq p$. By the homotopy lifting property we may assume that $q f=p$. It is well known that $\mathcal{E}$ must have the homotopy type of a $C W$ complex. (See, for example, [7, Lemma 3.2].) By applying the five lemma to the homotopy sequences ${ }^{7}$ of $p: E \rightarrow B$ and $q: \mathcal{E} \rightarrow B$ we conclude that $f$ is a homotopy equivalence.

So returning to the proof of Lemma 10.4 we have the task of building $p: E \rightarrow B$ and $f: E \rightarrow \mathcal{E}$. As is usual the following special case suffices for both the compact and noncompact cases. Let $\Delta^{n}$ be the standard $n$-simplex and assume that $p$ : $E \rightarrow \partial \Delta$ is a block bundle with fiber $F$. Also let $f: E \rightarrow \partial \Delta \times F$ be a block preserving map which is a homotopy equivalence over the vertices. We want to extend $p$ to a block bundle $\tilde{p}: \tilde{E} \rightarrow \Delta$ and extend $f$ to a map $\tilde{f}: E \rightarrow \Delta \times F$. (We lose no generality by using proj: $\Delta \times F \rightarrow \Delta$ in place of a Hurewicz fibration over $\Delta$.)

Since $f: E \rightarrow \partial \Delta \times F$ is a homotopy equivalence over the vertices, $f$ must also be a homotopy equivalence over the simplices. By inductively applying the Classification Theorem and $Z$-set unknotting theorem of [6] over the skeleta of $\partial \Delta$ we conclude that $f$ is homotopic to a block preserving homeomorphism $h: E \rightarrow$ $\partial \Delta \times F$. (It is here that the condition $\mathrm{Wh}(F)=0$ is invoked.) Let $E_{1}=E \times$ $[0,1] \amalg(\partial \Delta \times F) / \sim$, where $\sim$ is the minimal equivalence relation determined by $(x, 1) \sim h(x)$. This is just the mapping cylinder of $h$, where $E \equiv E \times\{0\}$ is the top

\footnotetext{
${ }^{7}$ The homotopy sequence for approximate fibrations is established in [9]
} 
and $\partial \Delta \times F$ is the base. Now define $\tilde{E}=E_{1} \cup(\Delta \times F)$, where $E_{1} \cap(\Delta \times F)=\partial \Delta$ $\times F$ is the base of $E_{1}$. Then $p: E \rightarrow \partial \Delta$ clearly extends to a block bundle $\tilde{p}: \tilde{E} \rightarrow \Delta$. If we write $\Delta \times F=[(\partial \Delta \times F) \times[0,1]] \cup(\Delta \times F)$, then it is easy to see how $f$ extends to $\tilde{f}: \tilde{E} \rightarrow \Delta \times F$.

Proof of Theorem 4. We are given a proper map $f: M \rightarrow B$ which is a $\beta$-fibration, for $\beta$ a fine open cover of $B$. Let $q: \mathcal{E} \rightarrow B$ be a Hurewicz fibration for which there is a homotopy equivalence $h: M \rightarrow \mathcal{E}$ so that $q h \simeq f$. By the homotopy lifting property we may assume that $q h=f$. It follows from Proposition 2.3 that $h$ is a $q^{-1}\left(\beta_{1}\right)$-equivalence, where $\beta_{1}$ is a fine open cover of $B$. As in the proof of Theorem 3 , the fiber of $\mathcal{E}$ (which is $\mathscr{F}$ ) is homotopy equivalent to a compact $Q$-manifold $F$. By Lemma 10.4 there is a block bundle $p: E \rightarrow B$ with fiber $F$ and a block-preserving homotopy equivalence $g: E \rightarrow \mathcal{E}$ which is block preserving. This can be done for any triangulation of $B$. If this triangulation is sufficiently fine, then $g: E \rightarrow \mathcal{E}$ is a $q^{-1}\left(\beta_{1}\right)$-equivalence. (Again we are invoking Proposition 2.3.)

Let $g_{1}: \mathcal{E} \rightarrow E$ be a $p^{-1}\left(\beta_{1}\right)$-inverse of $g$. Then $g_{1} h: M \rightarrow E$ is a map which fulfills the requirements of Theorem 3 , and so there is a homotopy of $g_{1} h$ to a homeomorphism $k: M \rightarrow E$. Moreover, the composition of $p$ with this homotopy is small in $B$. Then $p k: M \rightarrow B$ is a block bundle which is $\alpha$-homotopic to $f$.

\section{REFERENCES}

1. D. R. Anderson and W. C. Hsiang, The functors, $K_{-i}$ and pseudo-isotopies of polyhedra, Ann. of Math. (2) 105 (1977), 201-223.

2. H. Bass, Algebraic K-theory, Benjamin, New York, 1968.

3. T. A. Chapman, Cell-like mappings of Hilbert cube manifolds: Solution of a handle problem, General Topology Appl. 5 (1975), 123-145.

4. Homotopy conditions which detect simple homotopy equivalences, Pacific J. Math. (to appear).

5. appear). , Approximating maps into fiber bundles by homeomorphisms, Rocky Mountain J. Math. (to

6. __ Lectures on Hilbert cube manifolds, CBMS Regional Conf. Series in Math., No. 28 Amer. Math. Soc., Providence, R. I., 1976.

7. T. A. Chapman and Steve Ferry, Fibering Hilbert cube manifolds over ANRs, Compositio Math. 36 (1978), 7-35.

8. M. Cohen, A course in simple-homotopy theory, Springer-Verlag, New York, 1970.

9. D. Coram and P. Duvall, Approximate fibrations, preprint.

10. A. Dold, Partitions of unity in the theory of fibrations, Ann. of Math. (2) 78 (1963), 223-255.

11. R. D. Edwards and R. C. Kirby, Deformations of spaces of imbeddings, Ann. of Math. (2) 93 (1971), 63-88.

12. F. T. Farrell and W. C. Hsiang, A formula for $K_{1}\left(R_{\alpha}[T]\right)$, Proc. Sympos. Pure Math., vol. 17, Amer. Math. Soc., Providence, R. I., 1970.

13. Steve Ferry, Approximate fibrations with nonfinite fibers, Proc. Amer. Math. Soc. 64 (1977), 335-345.

14. The homeomorphism group of a compact Hilbert cube manifold is an ANR, Ann. of Math. (2) 106 (1977), 101-119.

15. M. Mather, Counting homotopy types of manifolds, Topology 4 (1965), 93-94.

16. L. C. Siebenmann, A total Whitehead torsion obstruction, Comment. Math. Helv. 45 (1970), 1-48.

17. E. H. Spanier, Algebraic topology, McGraw-Hill, New York, 1966.

18. C. T. C. Wall, Finiteness conditions for CW complexes, Ann. of Math. (2) 81 (1965), 55-69.

Department of Mathematics, University of Kentucky, LeXington, Kentucky 40506 